"This is the peer reviewed version of the following article: Chem. Rec. 2016, 1787-1806, which has been published in final form at http://onlinelibrary.wiley.com/doi/10.1002/tcr.201600030/abstract. This article may be used for non-commercial purposes in accordance with Wiley Terms and Conditions for Self-Archiving."

\title{
Enantioselective Vinylogous Organocascade Reactions
}

Hamish B. Hepburn, ${ }^{[a]}$ Luca Dell'Amico, ${ }^{[a]}$ and

Paolo Melchiorre ${ }^{*[a, b]}$

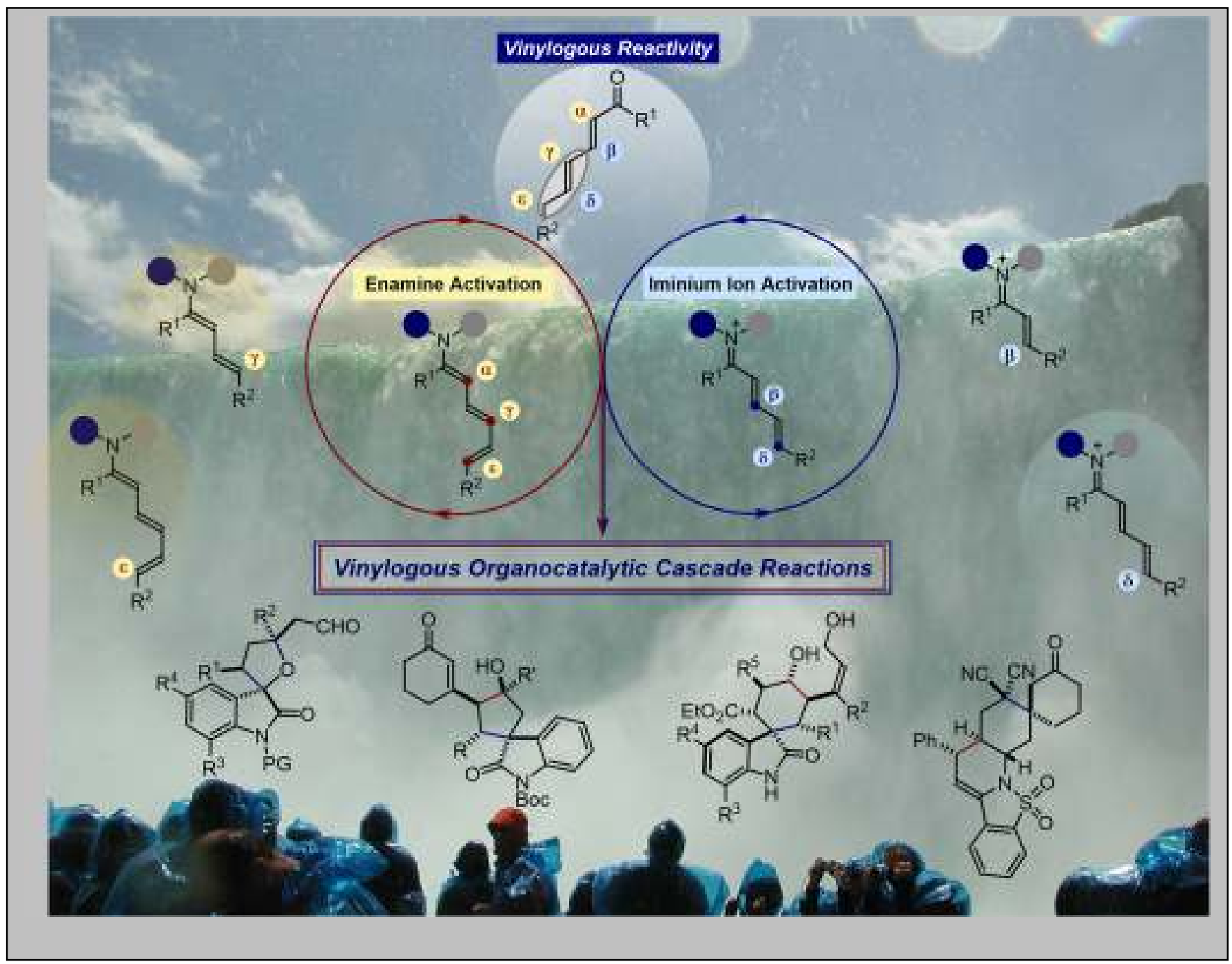




\begin{abstract}
Cascade reactions are powerful tools for rapidly assembling complex molecular architectures from readily available starting materials in a single synthetic operation. Their marriage with asymmetric organocatalysis has led to the development of novel techniques, which are now recognized as reliable strategies for the one-pot enantioselective synthesis of stereochemically dense molecules. In recent years, even more complex synthetic challenges have been addressed by applying the principle of vinylogy to the realm of organocascade catalysis. The key to success of vinylogous organocascade reactions is the unique ability of the chiral organocatalyst to transfer reactivity to a distal position without losing control on the stereo-determining events. This approach has greatly expanded the synthetic horizons of the field by providing the possibility of forging multiple stereocenters in remote positions from the catalyst's point of action with high selectivity while simultaneously constructing multiple new bonds. This article critically describes the developments achieved in the field of enantioselective vinylogous organocascade reactions, charting the ideas, the conceptual advances, and the milestone reactions that have been essential for reaching highly practical levels of synthetic efficiency.
\end{abstract}

\section{Introduction}

Cascade reactions are powerful tools for rapidly achieving molecular complexity since multiple chemical bonds are formed in a single synthetic operation. ${ }^{[1]}$ They fall in the broader definition of domino processes, ${ }^{[2]}$ where sequences of chemical transformations take place under the same reaction conditions (i.e. a single solvent, workup procedure, and purification step is required). Thus, all the reagents and catalysts are added at (or nearly at) the outset of the process to undergo a chemical transformation whose product becomes the substrate for the next step, whose product again becomes the substrate for the next step, and so on, until a product stable to the reaction conditions is reached. Cascade reactions pose an intellectually stimulating problem, since they require careful consideration of the compatibility of the transiently formed intermediates and the substrates, which should not undergo alternative irreversible reactions to form by-products. Thus, achieving a high degree of chemoselectivity represents the greatest obstacle in the design of cascade reactions. ${ }^{[3]}$ But success results in a big payoff, since cascade reactions reduce time-consuming and expensive protection/deprotection and isolation procedures of intermediates. In addition, this experimentally simple strategy offers the potential for rapidly increasing structural and stereochemical complexity. In a single synthetic step, it converts simple starting materials into complex molecular systems containing multiple stereocenters. Even more appealing is the development of enantioselective catalytic cascade reactions. ${ }^{[4]}$ By combining multiple asymmetric, catalytic transformations in a domino sequence, chemists can impart increased enantiomeric excess to the final product when compared to the corresponding discrete transformations. ${ }^{[5]}$ This requires a chiral catalyst that can effectively drive, in a

[a] Dr. L. Dell'Amico, Dr. H. B. Hepburn, and Prof. Dr. P. Melchiorre ICIQ - Institute of Chemical Research of Catalonia - the Barcelona Institute of Science and Technology

Avenida Països Catalans 16 - 43007, Tarragona, Spain

[b] Prof. Dr. P. Melchiorre

ICREA - Catalan Institution for Research and Advanced Studies, Passeig Lluís Companys 23 - 08010, Barcelona, Spain

E-mail: pmelchiorre@iciq.es

Homepage: http://www.iciq.org/research/research_group/prof-paolomelchiorre/ stereocontrolled fashion, consecutive catalytic reactions using different modes of substrate activation.

Given the above, it is easy to understand why the many synthetic benefits of cascade reactions have attracted the interest of asymmetric organocatalysis ${ }^{[6]}$ researchers. The stability, versatility, and compatibility with distinct functional groups make simple chiral organic catalysts perfectly suited for engineering effective asymmetric catalytic methods. The recent marriage with asymmetric organocatalysis has led to the emerging field of organocatalytic cascade reactions ${ }^{[7]}$, which has provided a way of achieving stereochemical and molecular complexity from readily available starting materials. The synthetic potential of this bioinspired approach has been validated by recent applications to the total synthesis of natural products. ${ }^{[8]}$ Within this context, a key role was played by chiral primary and secondary amines, which exploit fundamental and well-established mechanistic patterns, mainly using the chemistry of simple enamine ${ }^{[9]}$ and iminium ion intermediates ${ }^{[10]}$, to asymmetrically functionalize carbonyl compounds. ${ }^{[11]}$ Chiral amines were quickly recognized as ideal for catalyzing highly efficient cascade processes. This is mainly because of the ability of a single chiral amine to integrate orthogonal activation modes of carbonyl compounds into more elaborate reaction sequences, thus enabling the concomitant construction of two consecutive carbon-carbon bonds in one simple synthetic operation (Figure 1a).

To fully harness the synthetic power of organocascade catalysis, it was crucial to identify the iminium ion-enamine activation sequence as an enabling approach to highly efficient domino reactions (Figure 1b). ${ }^{[12]}$ The strategy is based on the 1,4-addition of a nucleophile $(\mathrm{Nu})$ to $\alpha, \beta$-unsaturated aldehydes or ketones and subsequent $\alpha$ functionalization of the resulting saturated carbonyl with an electrophile $(E I)$. In this well-defined sequence, the chiral amine plays an active role in both steps. Initially it lowers the lowest unoccupied molecular orbitals (LUMO-lowering activation) ${ }^{[13]}$ of chiral iminium ions, thus facilitating the $1,4-$ addition of a general nucleophile $(\mathrm{Nu})$, while in the second event it exerts an energy-raising effect on the highest occupied molecular orbital (HOMO-raising activation) of the enamine intermediate, promoting the addition to an electrophilic compound $(E I)$. The strategy has been extensively applied by the synthetic community to access $\alpha$ - and $\beta$-functionalized carbonyl chiral building blocks. ${ }^{[7,12]}$ 


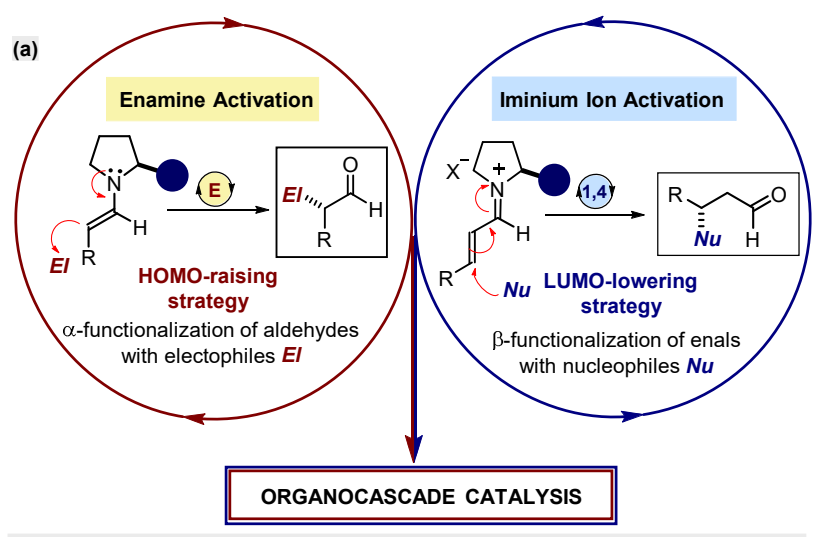

(b) Traditional approach
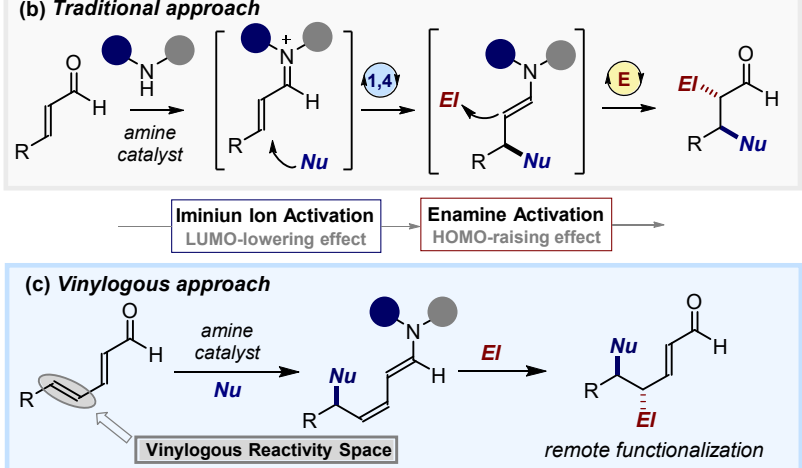

Figure 1. (a) Combining enamine and iminium ion activations. (b) The established iminium ion-enamine activation strategy for designing organocascade reactions. (c) Transferring the technique into a vinylogous reactivity pattern to forge multiple stereocenters remote from the catalyst's point of action; El: electrophile, Nu: nucleophile; E: Enamine activation; 1,4: iminium ion-mediated 1,4-addition.

Recently, the potential of organocascade catalysis has been further expanded to target more daunting synthetic objectives. Key to bringing this chemistry to the next level of efficiency and sophistication was the inclusion of vinylogous reactivity ${ }^{[14]}$ as a new design principle for developing unprecedented asymmetric organocatalytic domino reactions. This has facilitated the rapid synthesis of complex chiral molecules while selectively forging multiple stereocenters at distant positions from the catalyst's point of action (Figure 1c). ${ }^{[15]}$

Central to pursuing this goal is the ability of chiral amine catalysts to propagate the electronic effects inherent to aminocatalytic reactivity modes (i.e. the HOMO-raising and the LUMO-lowering activating effects) through the conjugated $\pi$-system of polyunsaturated carbonyls while transmitting the stereochemical information at distant positions. The fruitful combination of asymmetric aminocatalysis with the principle of vinylogy has expanded the chemists' ability to functionalize a carbonyl compound at distant positions such as the $\gamma-, \delta$-, and $\varepsilon$-carbon atoms (Figure 2). ${ }^{[16]}$

On the one hand, the propagation of the HOMO-raising electronic effect (inherent to enamine activation) through the conjugated $\pi-$ system of poly-unsaturated carbonyls has been used to induce vinylogous nucleophilicity in extended enamines. Dienamine $\left(E^{2}\right)^{[17]}$ and trienamine activations $\left(E^{3}\right)^{[18]}$ have accounted for the direct, stereoselective, and site-selective functionalization of unsaturated carbonyls at the $y$ and $\varepsilon$ positions, respectively. On the other hand, the successful combination of the LUMO-lowering effect (inherent to iminium ion activation) with the vinylogy principle accounts for the direct and stereoselective functionalization with nucleophiles of unmodified carbonyl compounds at the remote $\delta$-position (vinylogous iminium ion activation $(1,6)) \cdot{ }^{[19]}$

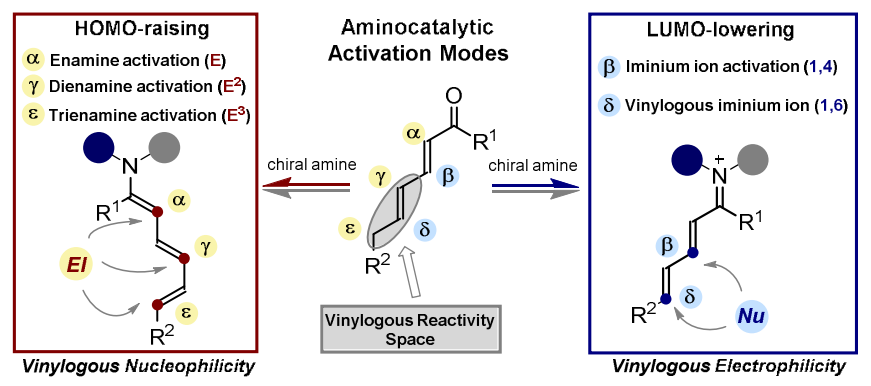

Figure 2. Established activation modes in aminocatalysis and the possibility of inducing vinylogous nucleophilicity and electrophilicity; the reactive carbon atoms are highlighted in different colors depending on their inherent reactivity: magenta stands for a nucleophilic position, blue for an electrophilic center; El: electrophile, Nu: nucleophile.

In this article, we discuss the many opportunities provided by the vinylogous aminocatalytic activation modes for the design of novel enantioselective organocascade reactions. The discussion is organized based on the nature of the initial vinylogous activation mode, which triggers the whole cascade sequence. After the condensation of polyunsaturated carbonyl compounds with a chiral amine catalyst, the HOMO-raising activation strategy brings about the formation of nucleophilic dienamine and trienamine intermediates, which can start a domino sequence upon reacting with an electrophile $\left(E^{2}\right.$ or $E^{3}$-initated cascade, respectively). The LUMO-lowering activation, instead, would provide the electrophilic vinylogous iminium ion that can initiate a cascade by reacting with a nucleophile (1,6-initated cascade). We have decided to include enantioselective vinylogous cycloaddition reactions only when evidence supporting a stepwise mechanism was provided in the original studies.

\section{HOMO-Raising Activation-Initiated Vinylogous Cascade Reactions}

\subsection{Sequential Dienamine-Iminium Ion Cascade Pathway}

The propagation of the HOMO-raising electronic effect through a conjugated $\pi$-system of a poly-unsaturated carbonyl compound (dienamine catalysis, $E^{2}$ ) has found extensive use in vinylogous cascade reactions. Upon the condensation of a chiral aminocatalyst with an $\alpha, \beta$-unsaturated carbonyl substrate, the transiently generated dienamine can attack a suitable electrophile to construct a new bond while leading to an electrophilic iminium ion (Figure 3). If an additional nucleophilic handle is present in the original substrate, a second intramolecular bond forming event can occur by means of an iminium ion-mediated conjugate addition $(1,4)$. This dienamine-iminium ion cascade sequence $\left(E^{2}-\right.$ $1,4)$ allows functionalization of a carbonyl compound at the $\gamma$ - and $\beta$-position and it requires the intervention of the aminocatalyst in both bond forming steps. 


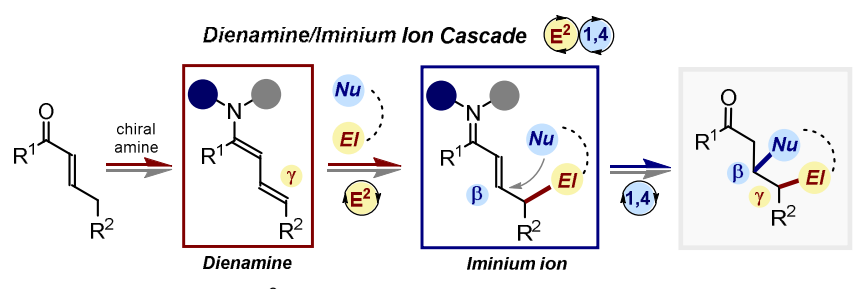

Figure 3. Dienamine $\left(\mathrm{E}^{2}\right)$ / iminium ion $(1,4)$ cascade sequence; the blue and grey circles represent the chiral scaffolds of the aminocatalyst.

The first dienamine-iminium ion activation strategy $\left(E^{2}-1,4\right.$ sequence) was reported by Woggon and co-workers in 2008 in the total synthesis of $\alpha$-tocopherol (Scheme 1), one of the most significant members of the vitamin $E$ family. ${ }^{[20]}$ The process constructed the core of a-tocopherol through a three-step cascade, two of which were catalyzed by the chiral silyl prolinol aminocatalyst 4 (TES: triethylsilane).

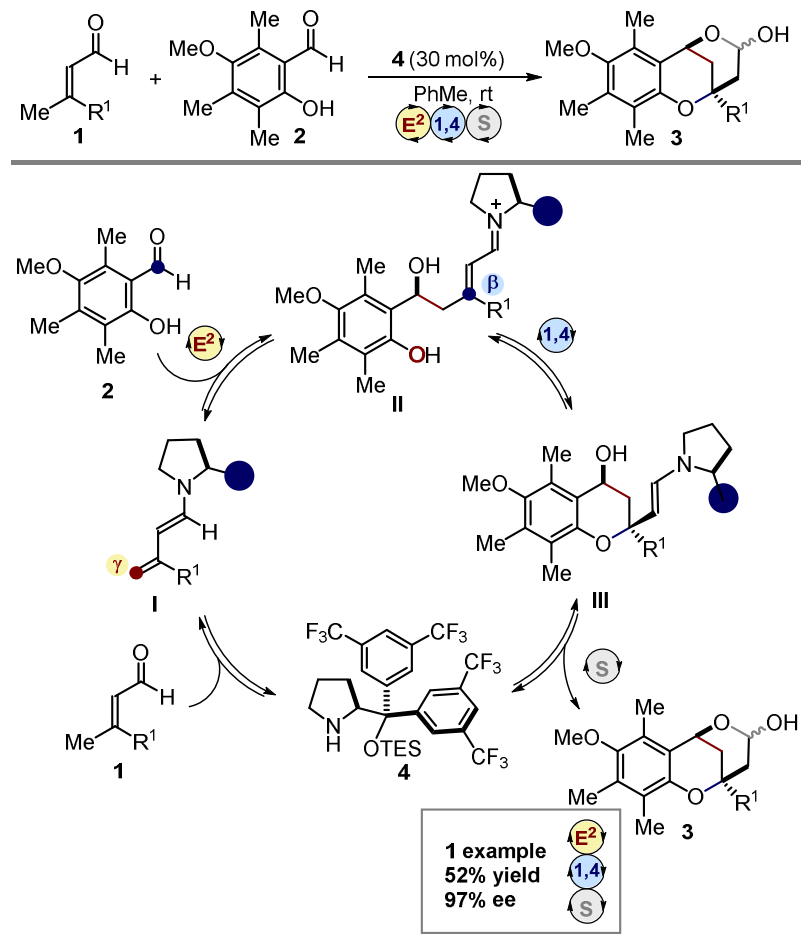

Scheme 1. The first example of a dienamine/iminium ion cascade; $E^{2}$. dienamine; 1,4: iminium ion; S: spontaneous step. The blue circle represents the chiral scaffolds of the aminocatalyst 4 . The reactive carbon atoms are highlighted in different colors depending on their inherent reactivity: magenta stands for a nucleophilic position, blue for an electrophilic center.

The reaction was triggered by the vinylogous aldol reaction between the terminal dienamine $\mathbf{I}$, formed upon catalyst condensation with the $\beta$-methyl substituted enal $\mathbf{1}$, and the salicyaldehyde derivative 2 . The resulting iminium ion intermediate II was then attacked by the phenolic oxygen in an oxa-Michael fashion to form a six-membered ring in III. Finally, following hydrolysis of the aminocatalyst, a third bond-forming step, namely a spontaneous $(S)$ intramolecular acetalization, led to the formation of $\mathbf{3}$ with high stereofidelity, an advanced intermediate en route toward the synthesis of a-tocopherol. Following this preliminary report, Woggon and others have since utilized this vinylogous triple cascade process to synthesize an array of related natural products. ${ }^{[21]}$

The dienamine-iminium ion cascade pathway has also served for the rapid construction of six-membered rings (Scheme 2). Jørgensen and co-workers reported that the reaction of enals $\mathbf{5}$ with $\beta, \gamma$-unsaturated- $\alpha$-ketoester $\mathbf{6}$, proceeding through a vinylogous 1,4-addition/oxa-Michael sequence, provided dihydropyran derivatives 7 in good yields (42-82\%) and enantioselectivities $\left(75-92 \%\right.$ ee). ${ }^{[22]}$ Crucial for the successful development of the chemistry was the design of a bifunctional secondary amine-thiourea catalyst that combined hydrogen $(\mathrm{H})$ bond directing activation of the electrophile with the dienamine activation of enals to ensure high levels of enantio- and regioselectivity. The bifunctional catalyst 8 was designed to facilitate the simultaneous activation of both $\alpha$-ketoester $\mathbf{6}$ and enal $\mathbf{5}$ while positioning them in an advantageous three-dimensional assembly to ensure control over the trajectory of the approaching electrophile. This secured high levels of stereoinduction in the addition of the dienamine IV to the conjugate acceptor $\mathbf{6}$. The resulting intermediate $\mathbf{V}$, which featured both an iminium ion and an enolate functionality, eventually underwent a ring closure through an oxa-Michael reaction to furnish dihydropyrans 7 . Although this reaction can be considered as an inverse-electrondemand Diels-Alder reaction, theoretical calculations supported a stepwise process as the more likely pathway. ${ }^{[22]}$

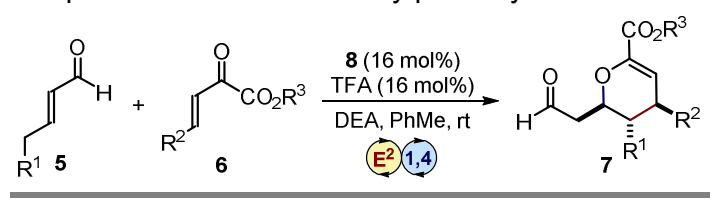

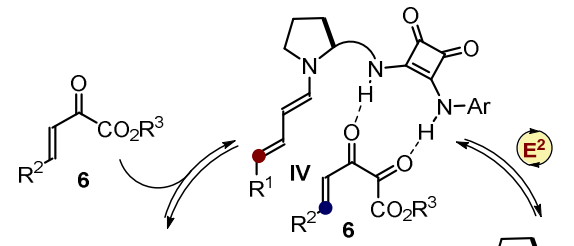

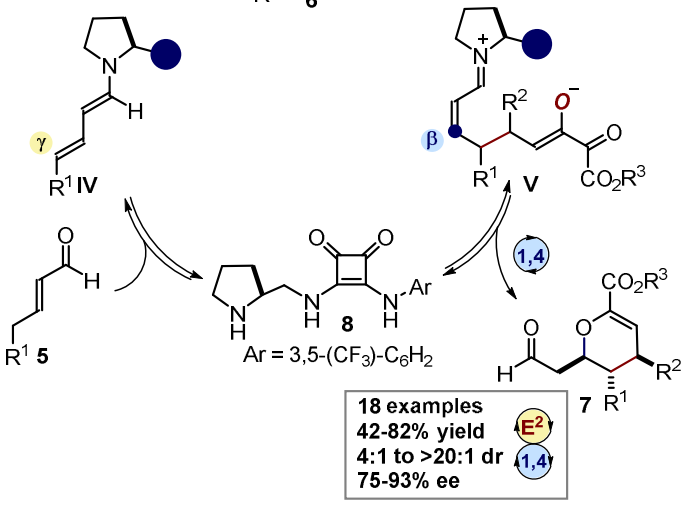

Scheme 2. Synthesis of dihydropyrans 7 through a $E^{2}-1,4$ cascade reaction facilitated by the bifunctional catalyst 8 . $E^{2}$ : dienamine; 1,4 : iminium ion; the blue circle in IV and $\mathbf{V}$ represents the chiral scaffolds of catalyst $\mathbf{8}$. The reactive carbon atoms are highlighted in different colors depending on their inherent reactivity: magenta stands for a nucleophilic position, blue for an electrophilic center. DEA: diethylamine; TFA: trifluroacetic acid.

As for the atom connectivity, the $E^{2}-1,4$ cascade sequence requires the enal substrate to act as a two carbon partner, with both new carbon-carbon bonds being formed in a 1,2-relationship. 
This means that, as seen in the previously discussed works (Schemes 1 and 2), the use of the four-atom partners 2 and 6 brings about the formation of six-membered ring products $\mathbf{3}$ and 7, respectively, through a formal [4+2] reaction. However, cyclic products of different ring size can be prepared by purposely modulating the reacting partners. For example, a $E^{2}-1,4$ cascade strategy to form a four-membered ring through a formal [2+2] reaction can be envisaged when using a two carbon partner, such as nitroolefins 9 (Scheme 3 ). These compounds can act similarly to $\alpha, \beta$-unsaturated carbonyl compounds $\mathbf{6}$, but the enolate emerging from the dienamine-triggered Michael addition is unlikely to act as oxygen-centered nucleophile. Independently and concurrently, the group of Jørgensen ${ }^{[23]}$ and Vicario ${ }^{[24]}$ developed vinylogous $E^{2}-1,4$ cascade sequences that utilized nitroolefins to facilitate the formation of enantioenriched cyclobutanes.

In analogy to the cascade depicted in Scheme 2, Jørgensen used the bifunctional secondary amine-thiourea catalyst 8 to activate the nitroolefins 9 while ensuring a close proximity with the dienamine IV (Scheme 3). ${ }^{[23]}$ The simultaneous activation of the two reacting partners secured a high stereofidelity in the first bond forming step (dienamine-driven conjugate addition) leading to the iminium ion intermediate VII. The nascent carbon-centered nucleophile then underwent an iminium ion-mediated conjugate addition to form cyclobutane 10 in good yields (62-93\%) and perfect stereoselectivities (single diastereoisomer and $99 \%$ ee or greater).

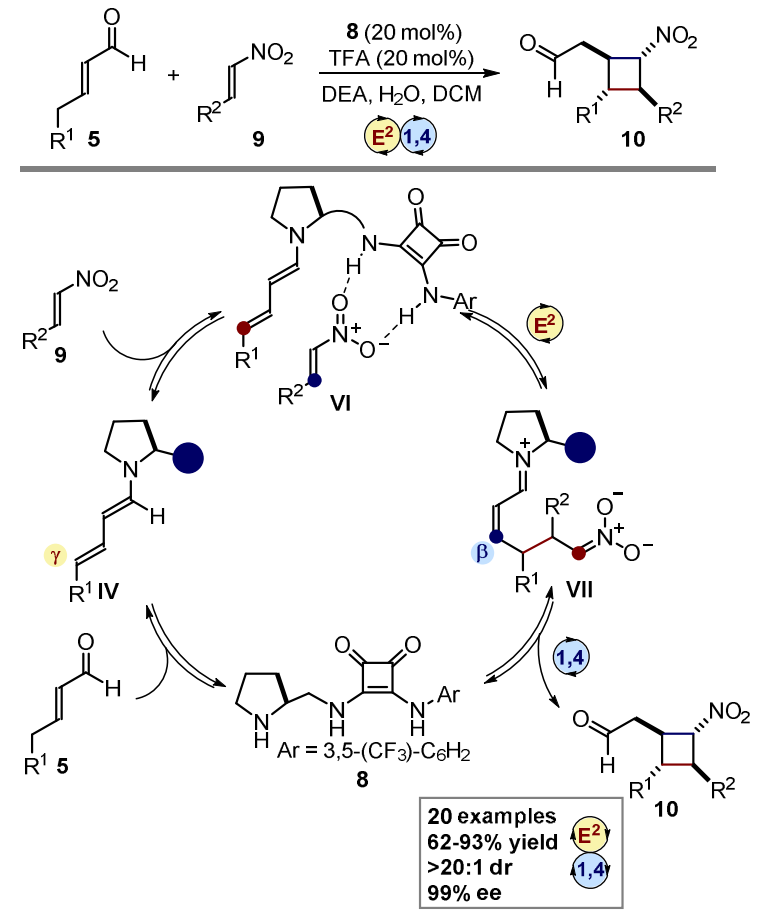

Scheme 3. $E^{2}-1,4$ cascade reaction: use of a two-carbon electrophile 9 to access cyclobutanes 10 by means of a vinylogous formal [2+2] reaction. $E^{2}$ : dienamine; 1,4 : iminium ion; the blue circle represents the chiral scaffold of catalyst 8 . DEA: diethylamine; TFA: trifluroacetic acid.

A related but different approach was reported by Vicario and coworkers ${ }^{[24]}$ who used two distinct organocatalysts to mimic the action of the bifunctional catalyst $\mathbf{8}$. Specifically, while the secondary amine 13 served to activate enal $\mathbf{5}$ through dienamine catalysis, the achiral thiourea catalyst $\mathbf{1 4}$ activated the nitroolefin 11 through $\mathrm{H}$-bonding catalysis (Scheme 4). Although intermediate VIII does not feature the same level of threedimensional organization achieved by a bifunctional catalyst, impressive levels of regio- and stereo-selectivity (85-95\% ee) were achieved. Following the attack of the dienamine IV on the activated nitroolefin in VIII, the iminium ion intermediate IX is formed, which then drives the conjugate addition of the enolate in an intramolecular fashion. Finally, the hydrolysis of the aminocatalyst 13 (TMS: trimethylsilyl), and a spontaneous acetalization (S), furnishes the bicyclic product 12.

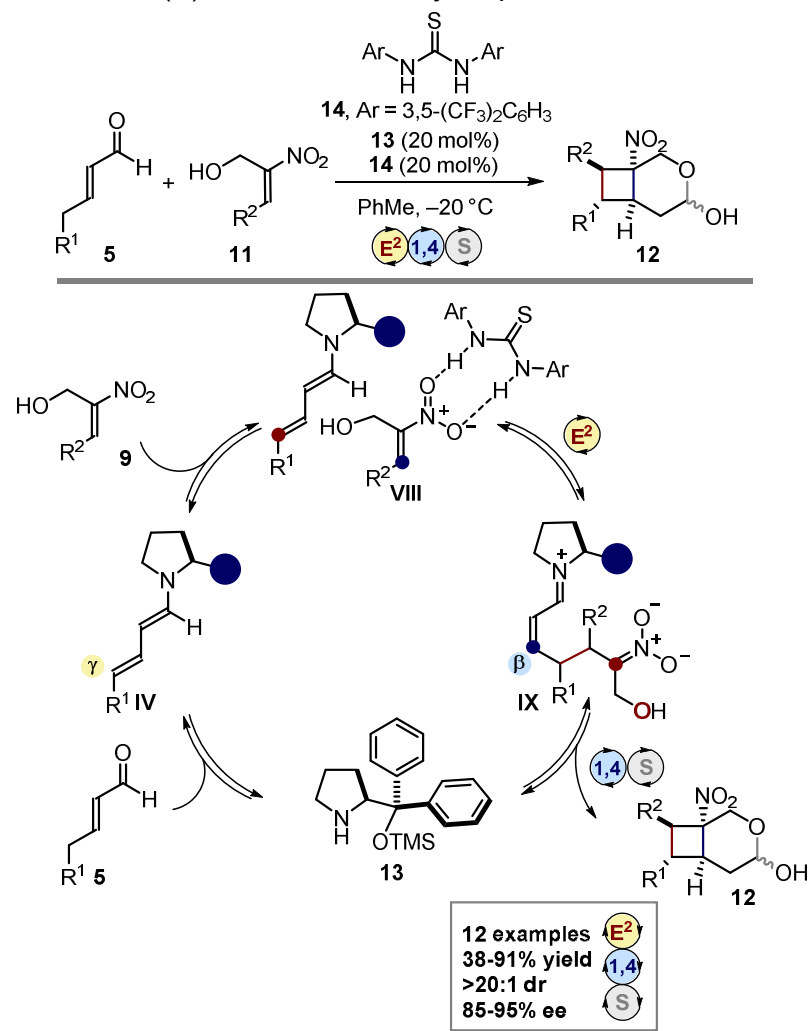

Scheme 4. $E^{2}-1,4$ cascade reaction: synthesis of enantioenriched cyclobutanes using two distinct organocatalysts. $E^{2}$ : dienamine; 1,4: iminium ion; $S$ : spontaneous step; the blue circle represents the chiral scaffold of catalyst 13 . The reactive carbon atoms are highlighted in different colors depending on their inherent reactivity: magenta stands for a nucleophilic position, blue for an electrophilic center.

\subsection{Sequential Dienamine-Enamine Cascade Pathway}

Vinylogous cascade reactions are not limited to functionalizing $\alpha, \beta$-unsaturated aldehydes in a 1,2-fashion. Unusual functionalizations in a 1,3-relationship, namely at the $\alpha$ - and $\gamma$ position, have also been achieved. This strategy relies on a dual HOMO-raising activation pathway which combines a dienamine/enamine sequence $\left(E^{2}-E\right.$, Figure 4). First, after condensation of a chiral aminocatalyst with an unsaturated carbonyl substrate, the resultant dienamine attacks an electrophile to form a transient iminium ion (not shown). However, $\mathrm{Y}$-deprotonation returns a nucleophilic dienamine which can trap a second electrophile at the a-position. This $E^{2}-E$ cascade reaction allows the functionalization of a carbonyl substrate at the 
$\mathrm{Y}$ - and $\alpha$ - positions and relies on the aminocatalyst for both bond forming steps.

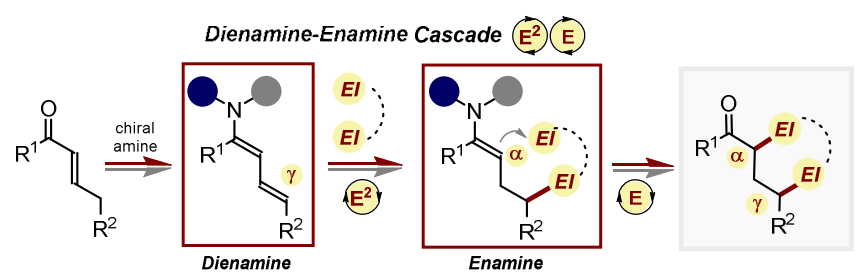

Figure 4. Dienamine $\left(\mathrm{E}^{2}\right)$ / enamine $(\mathrm{E})$ cascade sequence; the blue and grey circles represent the chiral scaffolds of the aminocatalyst.

The main issue to address when designing this cascade is that the reaction partner, reacting with the dienamine, should feature two distinct electrophilic sites. Thus, it is essential to discriminate between them to ensure high levels of regioselectivity. Recently the first $E^{2}-E$ cascade has been realized using enal 14 and a 2acetoxymethyl nitroolefin 15 (Scheme 5). ${ }^{[25]}$
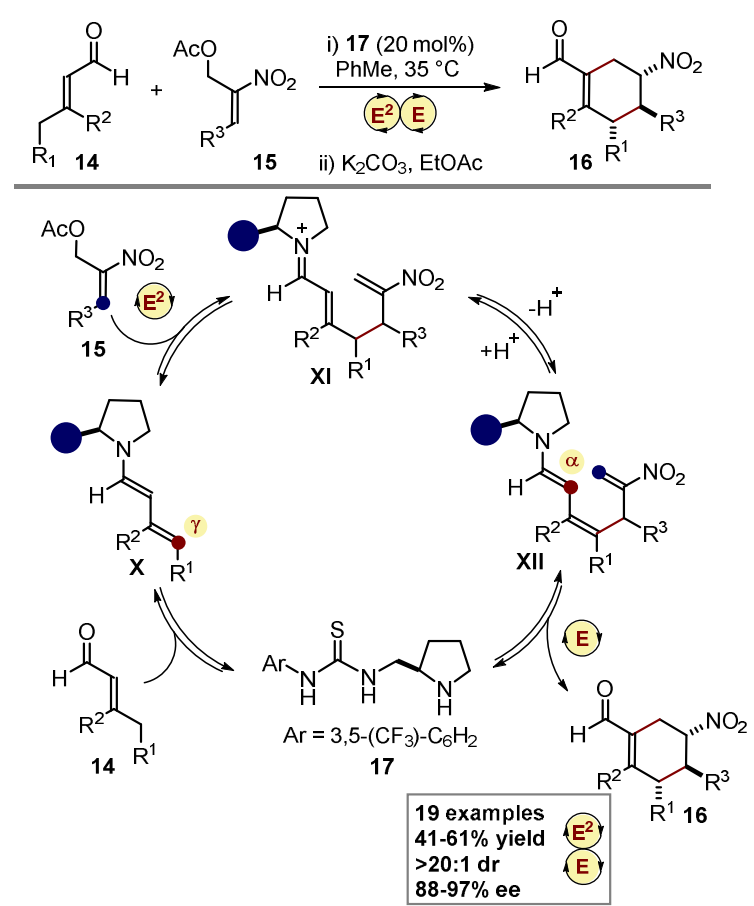

Scheme 5. Functionalization of the $\alpha$ - and $\gamma$ - position of enals 14 through a dienamine $\left(E^{2}\right)$ / enamine $(E)$ cascade sequence. $E^{2}$ : dienamine; $E$ : enamine; the blue circle represents the chiral scaffold of the bifunctional catalyst 17 . The reactive carbon atoms are highlighted in different colors depending on their inherent reactivity: magenta stands for a nucleophilic position, blue for an electrophilic center

The substrate 15 avoids the issue of regioselectivity since the second electrophilic position is revealed only once the initial dienamine-triggered reaction has happened. Indeed, the first conjugate addition of the dienamine $X$ to 15 , proceeding through an $S_{N} 2$ ' pathway, efficiently generates the required second electron-poor alkene moiety in XI through the elimination of acetic acid. Deprotonation to form XII favours the subsequent enaminemediated intramolecular conjugate addition ( $\alpha$-functionalization) to furnish the cyclohexene structures 16 in good yields (41-61\%) and high enantiomeric excess $(88-97 \%$ ee). The generally poor diastereomeric ratio of the process (about 2:1 dr) is ascribable to the low stereoselectivity of the protonation step in XII, which sets the stereocenter bearing the $\mathrm{R}^{1}$ substituent. However, it was found that treating the products 16 with $\mathrm{K}_{2} \mathrm{CO}_{3}$ readily epimerized the stereocenter to exclusively afford the trans-adduct.

\subsection{Sequential \\ Dienamine-Spontaneous \\ Cyclization}

\section{Cascade Pathway}

An alternative strategy to developing vinylogous cascade reactions is based on the use of a chiral aminocatalyst to only facilitate the formation of the first new bond, while a second spontaneous bond-forming step sequentially occurs (Figure 5). After condensation of an aminocatalyst with an unsaturated carbonyl substrate, the resultant dienamine attacks an electrophile (which can then reveal a nucleophilic moiety) to form the iminium ion intermediate. However, rather than an additional bond forming step at this point, hydrolysis of the catalysts creates a tethered carbonyl intermediate, which can then undergo a range of different spontaneous, non-catalyzed ring closing reactions to complete the cascade sequence $\left(E^{2}-S\right)$. Although this strategy does not involve the chiral aminocatalyst for both bond-forming steps, the stereochemical information encoded during the first dienamine step ensures high stereofidelity in the second spontaneous reaction, leading to products with high levels of stereocontrol. This approach lacks the complementary activation seen in the $E^{2}-1,4$ and $E^{2}-E$ cascades but it is still a powerful synthetic tool for the construction of multiple bonds in one step.

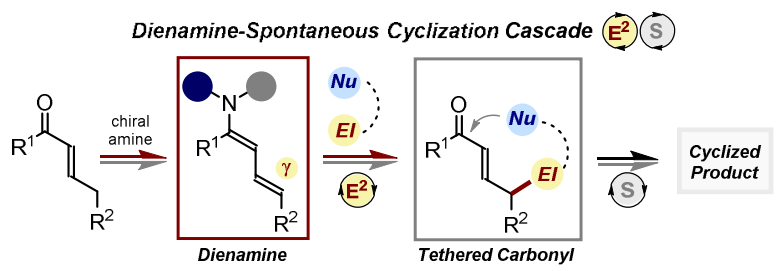

Figure 5. Dienamine $\left(\mathrm{E}^{2}\right) /$ spontaneous $(\mathrm{S})$ non-catalyzed cyclization cascade pathway; the blue and grey circles represent the chiral scaffolds of the aminocatalyst.

Given the presence of an aldehydic moiety in the products of aminocatalytic vinylogous cascades, the most commonly reported spontaneous step is an intramolecular aldol reaction. The first vinylogous cascade of this type involved the reaction of the enal 14 with a diene 18 to form tetracyclic skeletons 19 (Scheme 6). ${ }^{[26]}$ The mechanism is proposed to proceed as follows: the dienamine $\mathbf{X}$ participates in an inverse electron demand Diels-Alder reaction with 18 (this step can also be envisaged as a $E^{2}-1,4$ reaction sequence). After hydrolysis of the chiral aminocatalyst 13, isomerization and deprotonation of the newly formed intermediate XIII generates the enolate XIV, which undergoes an intramolecular aldol reaction to form product 19 in high yields (36$92 \%)$ and enantiomeric excess ( $80-98 \%$ ee). 


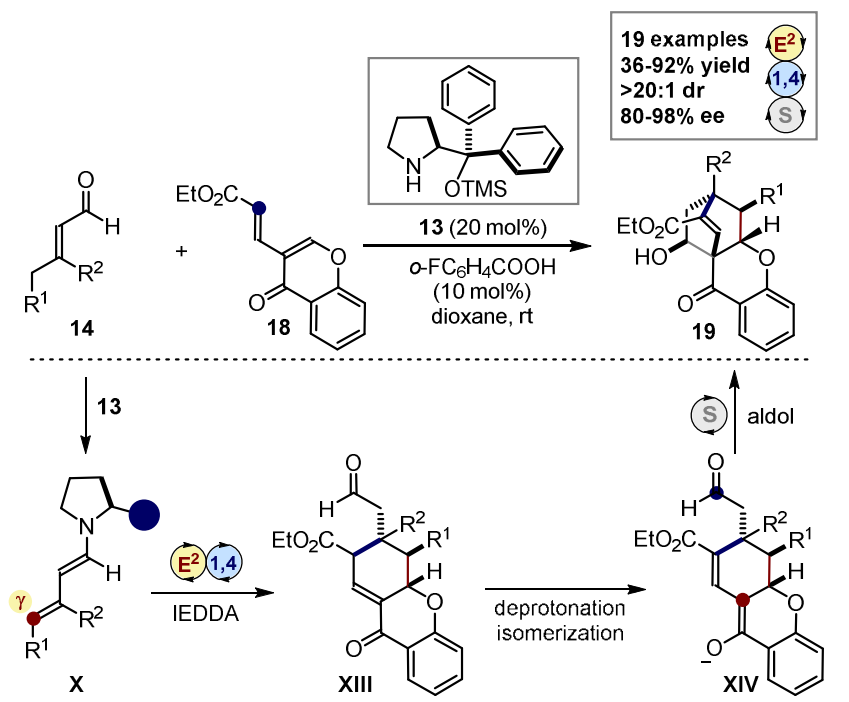

Scheme 6. Dienamine-spontaneous cyclization $\left(E^{2}-S\right)$ cascade sequence for the construction of tetracyclic products. The non-catalyzed step is an intramolecular aldol reaction; $E^{2}$ : dienamine; 1,4: iminium ion; $S$ : spontaneous step. The reactive carbon atoms are highlighted in different colors depending on their inherent reactivity: magenta stands for a nucleophilic position, blue for an electrophilic center.

The strategy of employing an aldol condensation in a $E^{2}-S$ cascade sequence was also used in conjunction with tetraenamine intermediates $\mathbf{X V}$ formed upon condensation of the aminocatalyst 23 with enal 20 (Scheme 7). ${ }^{[27]}$ Although these species have the potential to react at very remote positions, they were found to selectively react at the $y$-position. The reaction of $\mathbf{X V}$ with electrophilic 3-olefinic oxindoles $\mathbf{2 1}$ led to the formation of spirocyclic products 22 through a $E^{2}-S$ vinylogous cascade.

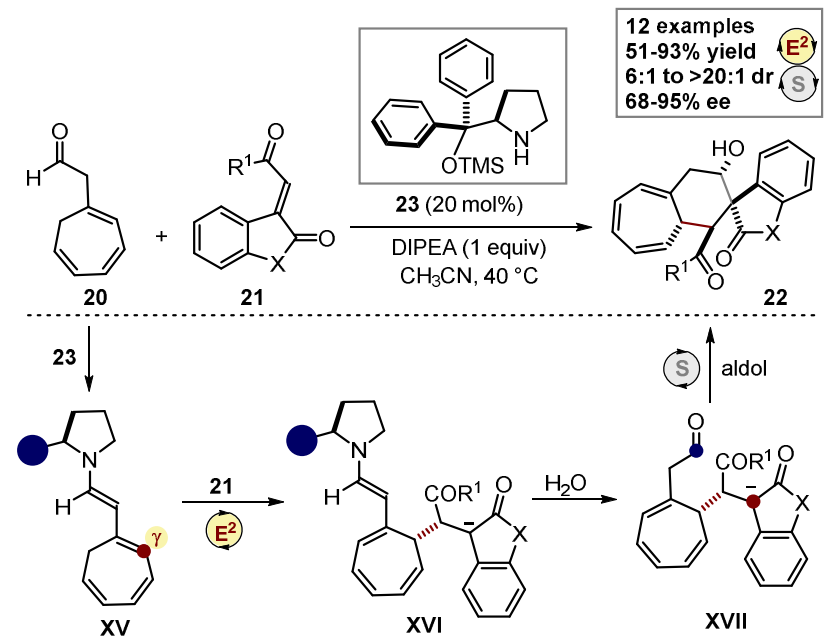

Scheme 7. Dienamine-spontaneous cyclization $\left(E^{2}-S\right)$ cascade sequence to form spirocyclic products. The non-catalyzed step is an intramolecular aldol reaction; $E^{2}$ : dienamine; $S$ : spontaneous step. The blue circle represents the chiral scaffolds of the aminocatalyst. The reactive carbon atoms are highlighted in different colors depending on their inherent reactivity: magenta stands for a nucleophilic position, blue for an electrophilic center. DIPEA: N,Ndiisopropylethylamine.

Mechanistically, the nucleophilic tetraenamine $\mathbf{X V}$ attacks the Michael acceptor 21. After hydrolysis of the aminocatalyst, the intermediate XVII, which contains both an aldehyde and an enolate, experiences an intramolecular aldol pathway to give $\mathbf{2 2}$ in good yields (51-93\%), diastereomeric ( $86: 14$ to $>20: 1)$, and enantiomeric excess $(68-95 \%$ ee).

$\mathrm{E}^{2}$-S cascade reactions often feature very similar intermediates to $E^{2}-1,4$ cascade sequences. The reasons why a reaction proceeds through a $E^{2}-S$ pathway instead can be subtle, and often ascribable to differences in the substrate's electronic and steric characteristics. For example, the cascade sequence illustrated in Scheme 8 appears, at first glance, very similar to the cascade illustrated in Scheme 3: it is indeed a reaction between an enal (23 or 5) and nitroolefin 9 in the presence of the bifunctional amine-thiourea catalyst $8{ }^{[28]}$ However, it the case of Scheme 3 the reaction proceeds through a $E^{2}-1,4$ cascade sequence to form the formal [2+2] cyclobutane product 10 while in Scheme 8 the reaction proceeds through a $E^{2}-S$ cascade affording the sixmembered ring 24. This divergent pathway can be attributed to the nature of the cyclic intermediate XIX. After nucleophilic attack of dienamine XVIII to nitroolefin 9, the $\beta$-position of the cyclic iminium ion $\mathbf{X I X}$ is not sufficiently electrophilic to permit the conjugate addition of the $\alpha$-nitro anion, due to the steric hindrance of the $\beta, \beta$-disubstitution pattern. This, along with the conformational constraints enforced by the cyclic structure of XIX, channels the process through a different pathway. Following hydrolysis of catalyst $\mathbf{8}$, the a-nitro anion undergoes a spontaneous Henry condensation, forming product 24 in moderate yields $(43-73 \%)$ and good enantiomeric excess (84$96 \%$ ee).

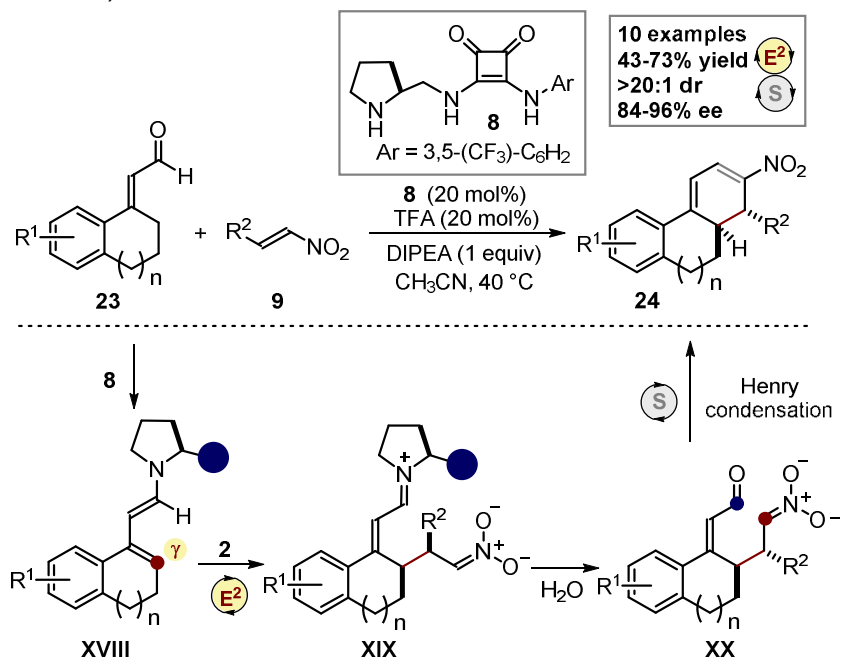

Scheme 8. Dienamine-spontaneous cyclization $\left(E^{2}-S\right)$ cascade between enal 23 and nitroolefin 9. The non-catalyzed step is an intramolecular Henry condensation; $\mathrm{E}^{2}$ : dienamine; $\mathrm{S}$ : spontaneous step. The blue circle represents the chiral scaffolds of the aminocatalyst. The reactive carbon atoms are highlighted in different colors depending on their inherent reactivity: magenta stands for a nucleophilic position, blue for an electrophilic center. DIPEA: N,Ndiisopropylethylamine.; TFA: trifluroacetic acid.

$\mathrm{E}^{2}$-S cascade reactions can also be utilized to rapidly construct structurally complex molecules through multiple bond forming events. This strategy served for the synthesis of bridged benzoxazocines 27 by means of a one-pot vinylogous cascade reaction (Scheme 9). ${ }^{[29]} \mathrm{A}$ multicomponent reaction between an aniline $\mathbf{2 5}$, a salicylaldehyde $\mathbf{2 6}$, and an enal 5 resulted in the formation of four new bonds and featured an unique $\mathrm{\gamma}, \bar{\delta}, i p s o-$ 
functionalization of enal $\mathbf{5}$. The reaction is proposed to proceed as follows: condensation of aniline $\mathbf{2 5}$ and salicylaldehyde $\mathbf{2 6}$ forms the aromatic imine $\mathbf{X X I}$, while condensation of the chiral aminocatalyst $\mathbf{2 8}$ and enal $\mathbf{5}$ forms the dienamine IV. These two intermediates undergo a stepwise Mannich-initiated formal aza[4+2] cyclization to form the cyclic six-membered intermediate XXII. Elimination of the aminocatalyst $\mathbf{2 8}$ renders the achiral iminium ion XXIII. Last, an oxa-Michael reaction furnishes the product 27 in good yield (22-91\%) and enantioselectivities (73$91 \%$ ee, Scheme 9).
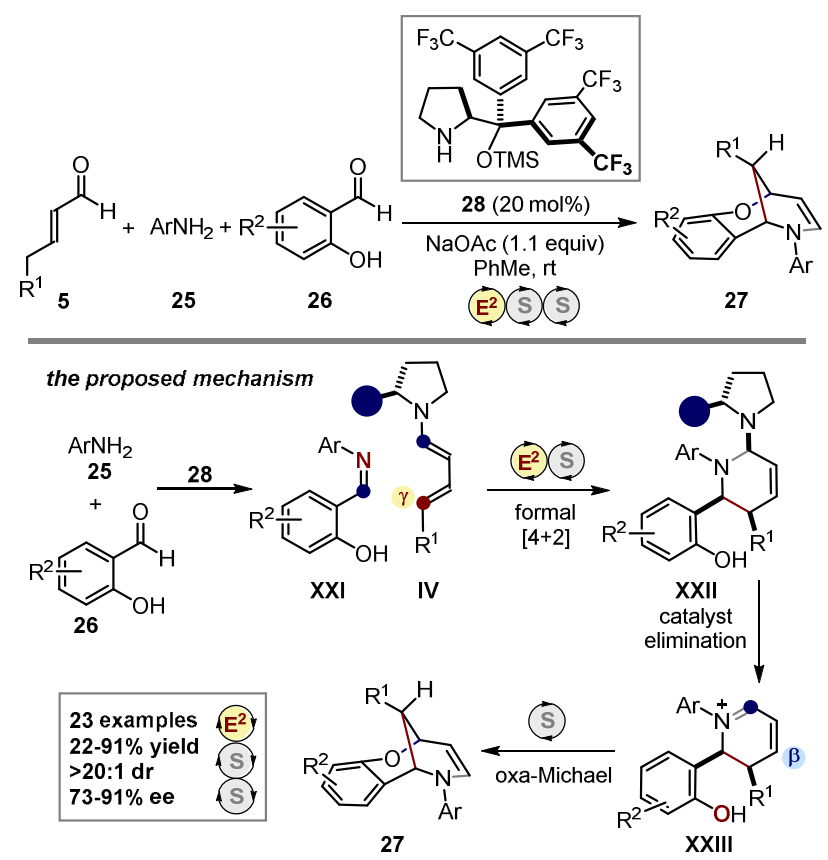

Scheme $\mathbf{9}$. The formation of structurally complex benzoxazocines $\mathbf{2 7}$ through a $\mathrm{E}^{2}$-S-S reaction sequence. The non-catalyzed step is an intramolecular oxaMichael reaction; $E^{2}$ : dienamine; $S$ : spontaneous step. The blue circle represents the chiral scaffolds of the aminocatalyst. The reactive carbon atoms are highlighted in different colors depending on their inherent reactivity: magenta stands for a nucleophilic position, blue for an electrophilic center.

\subsection{Extending the HOMO-Raising Activation Strategy Beyond the $y$-Position}

Condensation of an aminocatalysts with an $\alpha, \beta, \gamma, \delta$-unsaturated aldehyde forms a trienamine intermediate which can extend the HOMO-raising activation further into the vinylogous reactivity space, well beyond the $\mathrm{y}$ position. Trienamines feature similar reactivity profiles as the related enamine and dienamines and can facilitate functionalization at $\varepsilon$ carbon atom. ${ }^{[16 a]}$ Although the field of vinylogous cascade reactions using trienamine catalysis is far less developed than the corresponding dienamine field, some examples have been reported. These cascade sequences are based upon the trienamine/iminium ion $\left(E^{3}-1,4\right)$ pathway illustrated in Figure 6.

The earliest example of a $E^{3}-1,4$ cascade sequence was detailed in $2013^{[30]}$ with the trienamine-catalyzed aza-Diels-Alder process between the interrupted 2,5-dienones 28 and $\alpha, \beta$-unsaturated imines 29 in the presence of the cinchona-based primary amine catalyst $31^{[31]}$ (Scheme 10).

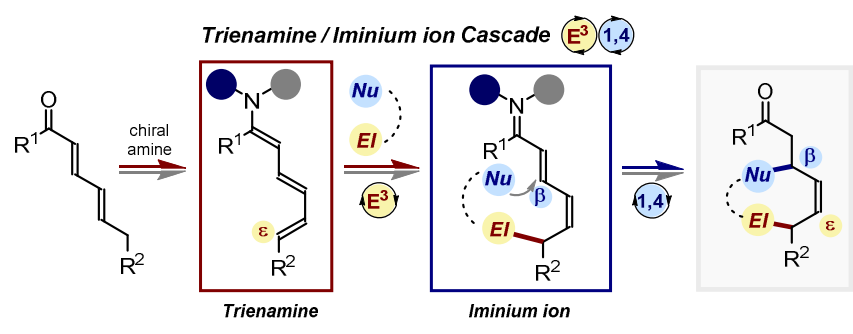

Figure 6. Trienamine $\left(\mathrm{E}^{3}\right)$ / iminium ion $(1,4)$ cascade sequence; initial HOMO raising activation event at the $\varepsilon$-position, followed by a complementary LUMOlowering event. The blue and grey circles represent the chiral scaffolds of the aminocatalyst.

It was found that tethering a Michael donor to the interrupted dienone 28 resulted in a $E^{3}-1,4$ cascade sequence to generate spirocycle $\mathbf{3 0}$ in $92 \%$ yield, $99 \%$ ee, and as a single diastereomer. The trienamine intermediate XXIV was formed through condensation of the cinchona-based primary amine catalyst $\mathbf{3 1}$ with skipped dienone $\mathbf{2 8}$ and this intermediate served as diene for an inverse electron-demand aza-Diels-Alder with imine 29 to form the intermediate $\mathbf{X X V}$ (this step, if stepwise, can also be seen as a $E^{3}-1,6$ sequence). Deprotonation of the acidic proton in the malononitrile moiety resulted in an iminium ion-mediated addition to form the intermediate $\mathbf{X X V I}$, which after catalyst hydrolysis afforded the product 30 . ${ }^{[30]}$
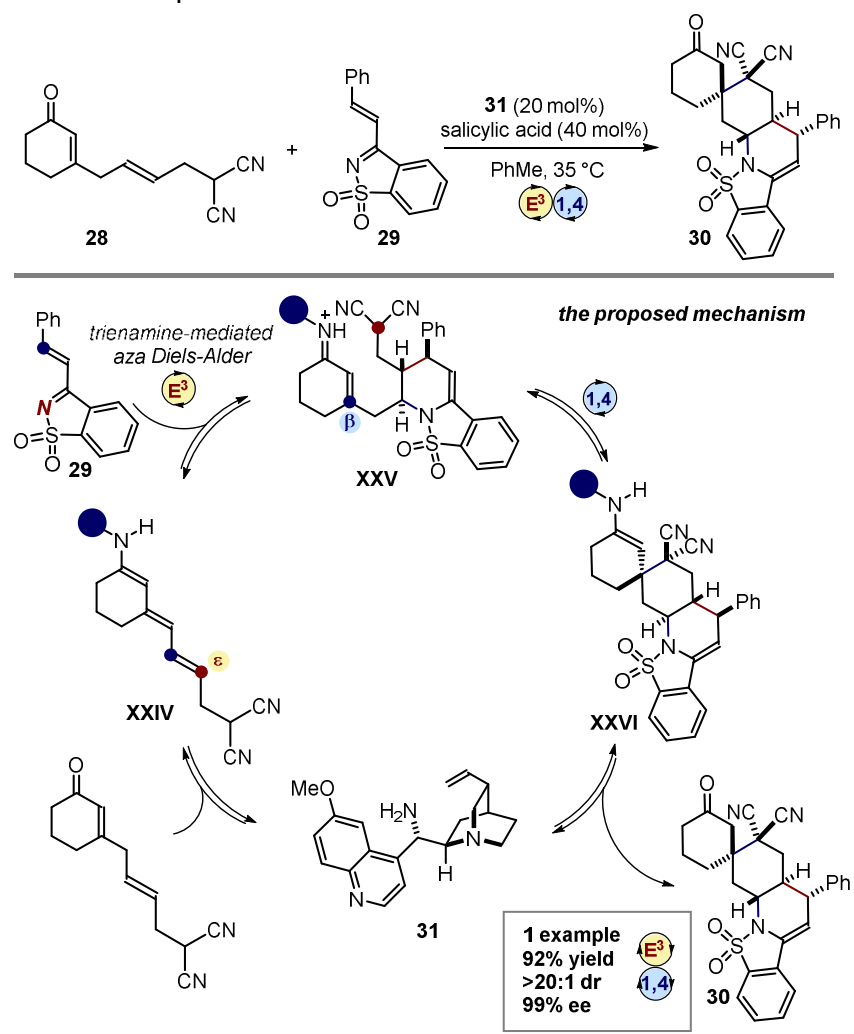

Scheme 10. The first trienamine-initiated vinylogous cascade reaction: $E^{3}-1,4$ reaction sequence; $E^{3}$ : trienamine; 1,4 : iminium ion. The blue circle represents the chiral scaffold of the cinchona-based primary amine catalyst 31 . The reactive carbon atoms are highlighted in different colors depending on their inherent reactivity: magenta stands for a nucleophilic position, blue for an electrophilic center. 
Very recently, the $E^{3}-1,4$ cascade strategy has been utilized in the formal [4+2] reaction between a nitroindole 33 and an $\alpha, \beta, \gamma, \delta-$ unsaturated aldehyde $\mathbf{3 2}$ catalyzed by a bifunctional catalyst 35 to furnish the tricyclic products 34 (Scheme 11). ${ }^{[32]}$ The condensation of the aminocatalyst $\mathbf{3 5}$ with enal $\mathbf{3 2}$ leads to the formation of the trienamine XXVII, while simultaneously the urea moiety within $\mathbf{3 5}$ activates the nitroolefin $\mathbf{3 3}$ through $\mathrm{H}$-bonding interaction. This double activation manifold promotes the trienamine-driven conjugate addition to form the iminium ion XXVIII. The resultant $\alpha$-nitro anion attacks the electrophilic iminium ion to form the intermediate XXIX. Finally, elimination of the nitro group and rearomatization of the indole nucleus leads to the formation of the product 34 in good yields $(51-77 \%)$ and enantioselectivities (49-98\% ee).
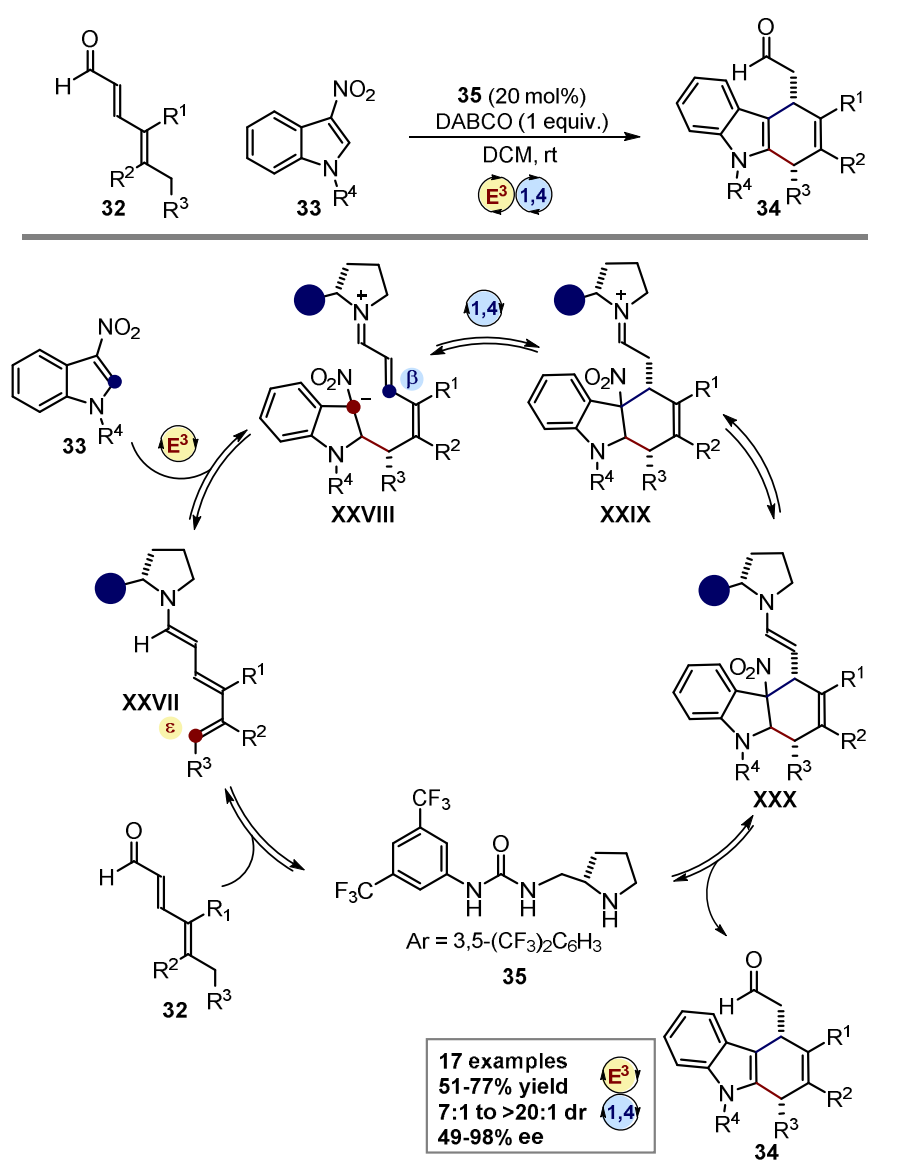

Scheme 11. The formation of cyclic products 34 through $E^{3}-1,4$ cascade reaction. $E^{3}$ : trienamine; 1,4 : iminium ion. The blue circle represents the chiral scaffold of the bifunctional amine-thiourea catalyst. The reactive carbon atoms are highlighted in different colors depending on their inherent reactivity: magenta stands for a nucleophilic position, blue for an electrophilic center.

It has also been possible to develop a trienamine-initiated vinylogous cascade reaction of the $\mathrm{E}^{3}$-S type. The use of the chiral secondary amine catalyst $\mathbf{1 3}$ triggered the formation of tricyclic benzo[de]quinolone products 38 from quinone imine ketals 36 and 2,4-dienals 37 through a $E^{3}$-S pathway in good yields (60$81 \%)$ and enantiomeric excess (85-98\% ee, Scheme 12$) \cdot{ }^{[33]}$ The cascade sequence is initiated by the condensation of catalyst 13 with enal $\mathbf{3 7}$ to form a reactive trienamine which then participates in a Diels-Alder reaction to furnish the [4+2] adduct $\mathbf{X X X I}$. Aromatization of this intermediate through elimination of an alcohol forms the quinolone core $\mathbf{X X X I I , ~ w h i c h ~ u n d e r g o e s ~ a ~ f i n a l ~}$ spontaneous cyclization. The resulting hemi-acetal (not shown in Scheme 12) is reduced in situ to aid isolation of the product 38.
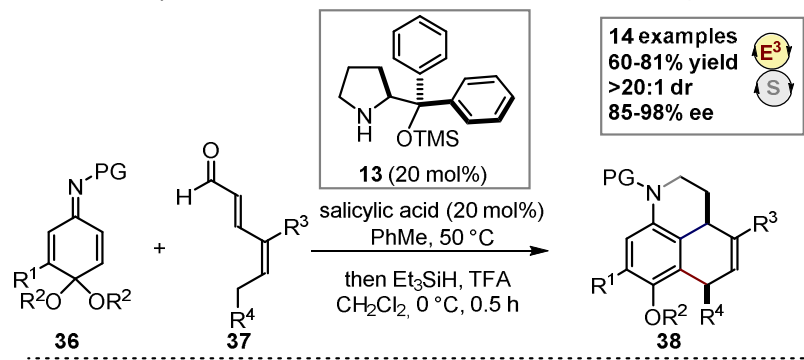
$>20: 1 \mathrm{dr}$ $85-98 \%$ ee

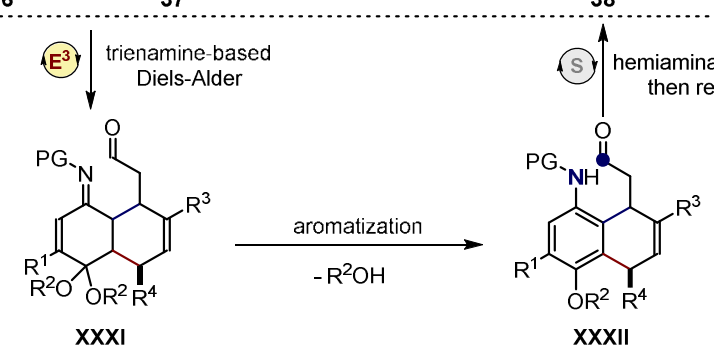

Scheme 12. $E^{3}-S$ cascade sequence to generate quinolones; $E^{3}$ : trienamine; $S$ : spontaneous step. The reactive carbon atoms are highlighted in different colors depending on their inherent reactivity: magenta stands for a nucleophilic position, blue for an electrophilic center.

\section{Vinylogous Iminium Ion-Initiated Cascade Reactions}

In 2012, our research group became interested in the possibility of transmitting the LUMO-lowering activating effect, inherent to iminium ion catalysis $(1,4)$, through the conjugated $\pi$-system of polyunsaturated carbonyl compounds, in order to realize a direct nucleophilic 1,6-addition. The difficulty of this goal becomes apparent when considering that the larger m-orbital LUMO coefficient and the more positive Mulliken atomic charge of extended iminium ions reside at the $\beta$ carbon atom, thus explaining the intrinsic preference for a nucleophilic 1,4-addition manifold. ${ }^{[19 b]}$ The requirement for the chiral catalyst to forge a remote stereocenter with high fidelity while inducing exclusive $\delta$ site selectivity in the 1,6-addition manifold further complicates matters. We have found, however, that when a cinchona-based primary amine catalyst condenses with $\beta$-substituted cyclic dienones, it determines the formation of an extended iminium ion intermediate (Figure 7), with an enhanced electrophilic character at the $\delta$-carbon atom. The resulting vinylogous iminium ion activation $(1,6)$ was used to develop an asymmetric organocatalytic 1,6-addition of alkyl thiols to cyclic $\alpha, \beta, \gamma, \delta$ unsaturated dienones 39 proceeding with high stereocontrol and $\delta$-site selectivity. ${ }^{[19 a]}$ Crucial for success was $i$ ) the unique ability of a cinchona amine of type $\mathbf{4 0}$ to stereochemically bias intermediary cyclic species thus ensuring highly predictable reaction outcomes along with ii) the inherent steric bias of the $\beta$ substituent in $\mathbf{3 9}$, which provided a useful steric element for securing $\delta$-site selectivity by suppressing the competing 1,4addition manifold. The development of the vinylogous iminium ion
の 
activation provided new opportunities for the design of novel cascade reactions.

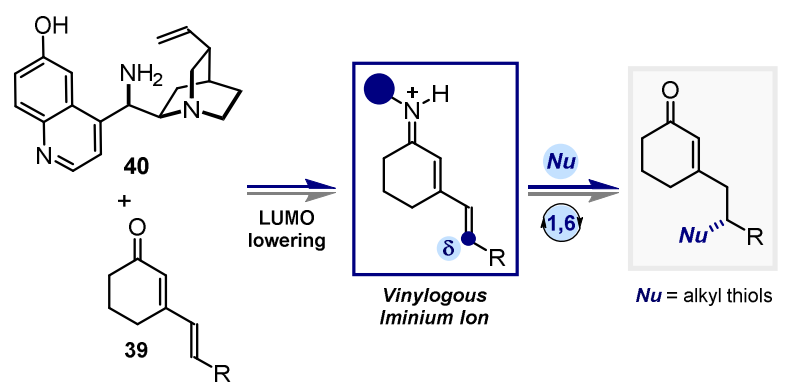

Figure 7. Asymmetric 1,6-additions to cyclic dienones 39 mediated by vinylogous iminium ion activation $(1,6)$. The blue circle represents the chiral scaffold of the cinchona-based primary amine catalyst.

\subsection{Sequential Vinylogous Iminium Ion/Dienamine Cascade Pathway}

The identification of the vinylogous iminium ion activation allowed the design of more complex cascade reactions upon combination with other aminocatalytic activation modes. For example, the condensation of the chiral aminocatalyst generates an extended iminium ion which can facilitate a nucleophilic attack at the $\delta$ position $(1,6)$. After this addition, a nucleophilic dienamine intermediate is transiently formed. If an electrophilic position is present in the original substrate, a second intramolecular bond forming event can occur under dienamine activation $\left(E^{2}\right.$, Figure 8). The vinylogous iminium ion-dienamine $\left(1,6-E^{2}\right)$ cascade accounts for the functionalization of a $\alpha, \beta, \gamma, \delta$-unsaturated carbonyl compound at the $\delta$ - and $y$-position while relying on the chiral aminocatalyst for both bond forming steps.

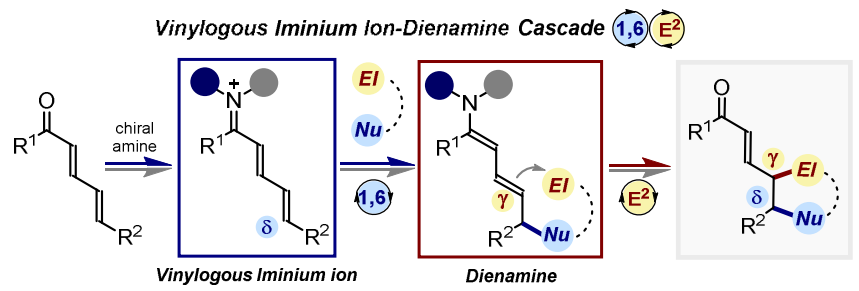

Figure 8. Vinylogous iminium ion $(1,6)$ /dienamine $\left(E^{2}\right)$ cascade pathway; the blue and grey circles represent the chiral scaffolds of the aminocatalyst.

The first example of 1,6- $E^{2}$ cascade capitalized upon the already discussed ability of the cinchona-based primary amine catalyst of type $\mathbf{4 0}$ to condense with $\beta$-substituted cyclic dienones $\mathbf{3 9}$ while controlling the geometry of the reactive cyclic iminium ion intermediate XXXIII. We recognized that a well-suited substrate, characterized by a dichotomous reactivity profile, was crucial to realizing a cascade process. On the basis of the inspiring studies by Carlos Barbas III, ${ }^{[34]}$ the 3-substituted oxindole 41 seemed well-tailored to first act as a carbon-centered nucleophile and then to develop, after the $\delta$-site 1,6-addition, an electrophilic behavior (Scheme 13). The pendant carbonyl moiety within the transiently generated nucleophilic dienamine intermediate XXXIV did drive an intramolecular aldol reaction, resulting in a fast cyclization. The product of the vinylogous cascade reaction, which is based on a $\delta$-addition/aldolization sequence, is a complex spirocyclopentane oxindole 42 bearing four contiguous stereocenters and a preserved $\alpha, \beta$-unsaturated carbonyl system. ${ }^{[35]}$

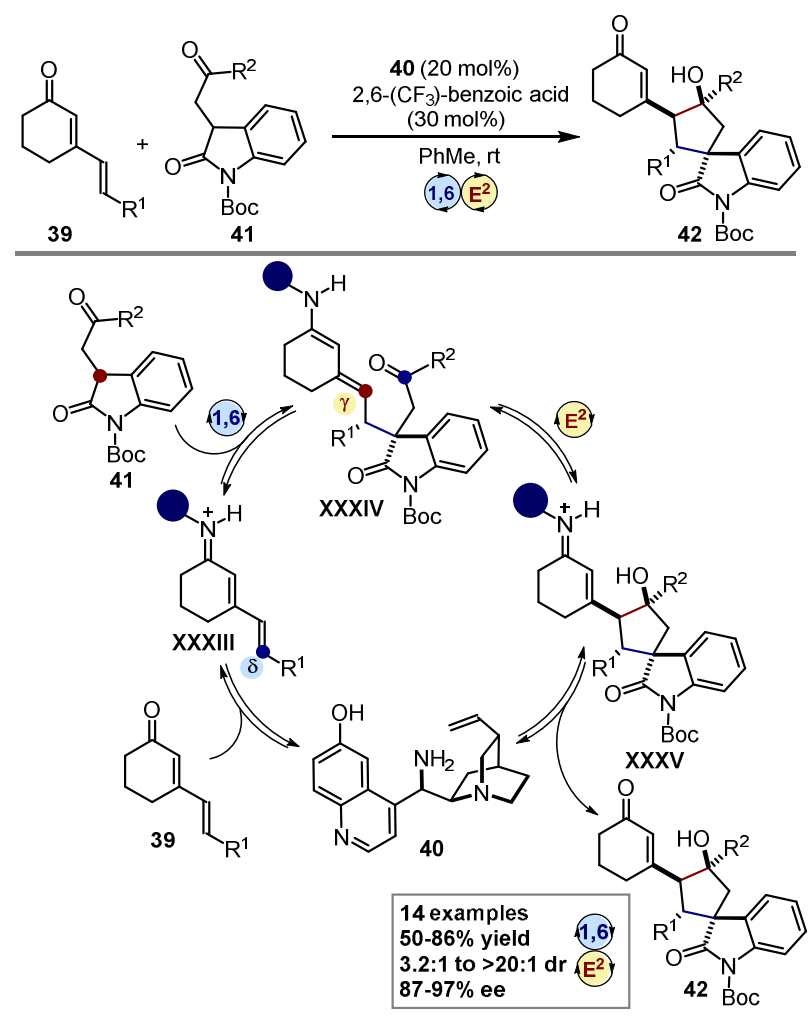

Scheme 13. The first report of a $1,6-E^{2}$ cascade sequence; 1,6 : vinylogous iminium ion; $E^{2}$ : dienamine. The blue circle represents the chiral scaffold of the cinchona-based primary amine catalyst. The reactive carbon atoms are highlighted in different colors depending on their inherent reactivity: magenta stands for a nucleophilic position, blue for an electrophilic center.

Building upon this precedent, Dixon, Ye and co-workers developed, in 2015, a vinylogous organocascade reaction employing five-membered cyclic dienones $\mathbf{4 3}$ and $\mathrm{N}$-protected pyrrolinones $44{ }^{[36]}$ The process, promoted by the chiral primary amine catalyst $\mathbf{4 6}$, furnished enantioenriched tricyclic $\gamma$-lactams 45 in moderate chemical yields $(37-67 \%)$ and diastereocontrol and a high level of enantiocontrol (92-99\% ee, Scheme 14). A non-trivial reaction mechanism was proposed: After the vinylogous iminium ion formation and the subsequent 1,6-addition reaction, an isomerization of the transient dienamine intermediate XXXVII takes place driven by the formation of the corresponding iminium ion intermediate (not shown). The newly formed internal dienamine $\mathbf{X X X V I I I ~ i s ~ n o w ~ w e l l ~ p o s i t i o n e d ~ t o ~ u n d e r g o ~ a n ~}$ intramolecular Michael addition forming the second carboncarbon bond. Migration of the C-C double bond in XXXIX to the other side of the carbonyl group (ascribable to an isomerization via the dienamine of cyclopentenone presumably driven by the thermodynamic stability of the product) furnished, after hydrolysis and liberation of the catalyst, the final tricyclic product 45. 


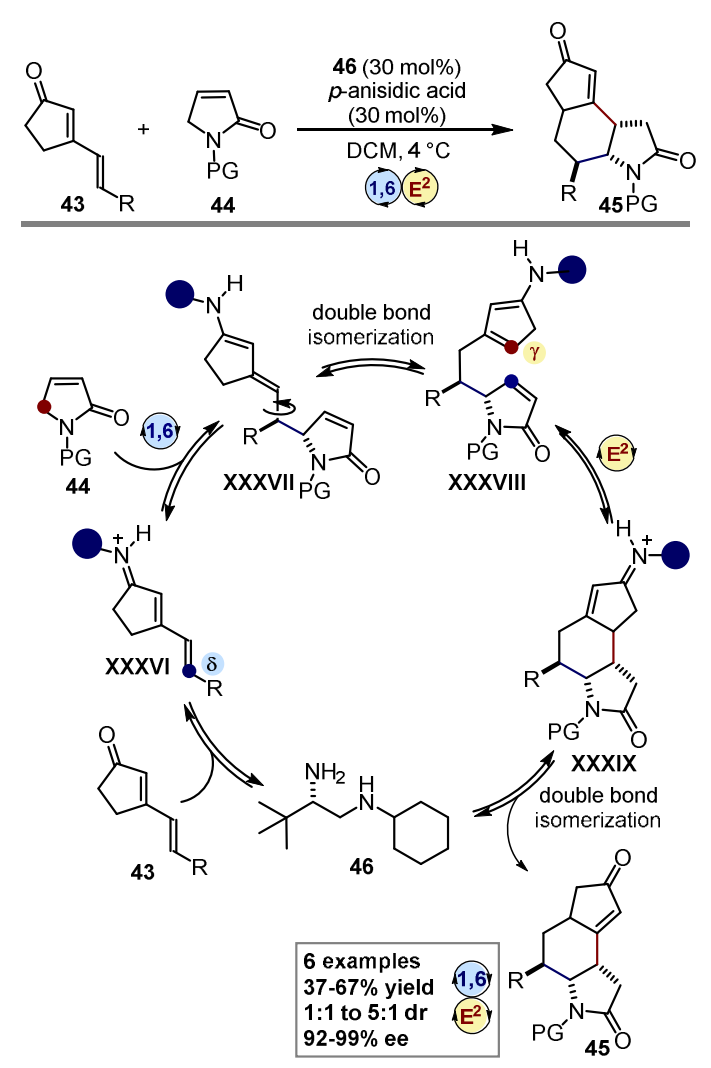

Scheme 14. Synthesis of tricyclic products 45 through a $1,6-E^{2}$ cascade reaction; 1,6: vinylogous iminium ion; $\mathrm{E}^{2}$ : dienamine. The blue circle represents the chiral scaffold of the aminocatalyst 46. PG: protecting group. The reactive carbon atoms are highlighted in different colors depending on their inherent reactivity: magenta stands for a nucleophilic position, blue for an electrophilic center.

The concept of vinylogous organocascade reaction based on 1,6addition/dienamine chemistry was then successfully extended from cyclic enone to cyclic enals. In 2013, Jørgensen and coworkers reported an enantioselective remote aziridination reaction, based on the 1,6- $E^{2}$ sequence. ${ }^{[37]}$ Extended cyclic 2,4dienals of type $\mathbf{4 7}$ formed the vinylogous iminium ion in situ which later underwent 1,6-addition from the nitrogen-centered nucleophile 48, resulting in the formation of the dienamine XLI. An intramolecular $S_{N} 2$ process, facilitated by the leaving group (TsO-), led to the aziridinated iminium ion XLII. Hydrolysis of XLII afforded the desired aziridine 49 in good yields, excellent diastereoselectivity, and moderate to excellent enantiocotrol (40$95 \%$ ee).

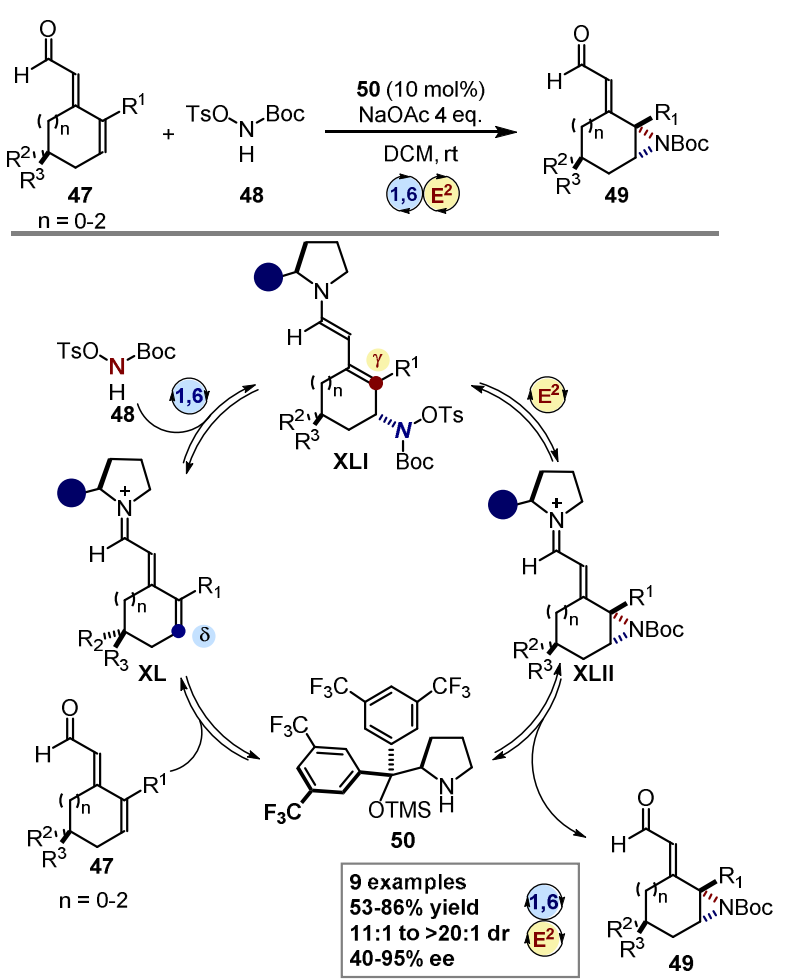

Scheme 15. Remote aziridination of cyclic enals by means of a $1,6-E^{2}$ cascade reaction; 1,6 : vinylogous iminium ion; $E^{2}$ : dienamine. The blue circle represents the chiral scaffold of the aminocatalyst $\mathbf{5 0}$. The reactive carbon atoms are highlighted in different colors depending on their inherent reactivity: magenta stands for a nucleophilic position, blue for an electrophilic center.

Although the 1,6- $E^{2}$ sequence demonstrated its usefulness for the implementation of vinylogous cascade strategies, as highlighted in Schemes 13-15, the synthetic potential of the approach was limited by the need for a cyclic substrate. Clearly, the use of a linear $\alpha, \beta, \gamma, \delta$-unsaturated carbonyl compound would provide a tool for designing more flexible and synthetically relevant vinylogous cascade reactions. However, the successful realization of this plan was far from easy since controlling the site and stereo-selectivity in the initial 1,6-addition reactions of linear substrates poses more than a conundrum. As a matter of fact, it is easier for a chiral amine catalyst to stereochemically bias the geometry of a cyclic vinylogous iminium ion intermediate than a linear counterpart. Such geometrical control is generally essential for securing selectivity. We found, though, that the presence of a steering group at the $\beta$-position of linear 2,4-dienals accounted for a perfect $\delta$-site selective 1,6 -addition reaction. This was critical to the design of a $1,6-E^{2}$ process proceeding by way of an aminocatalyzed 1,6-addition/vinylogous $S_{N} 2$ sequence (Scheme 16). The notion that a suitable reaction partner for the cascade requires the ability of the substrate of acting as both a nucleophile and an electrophile suggested 3-chlorooxindole $\mathbf{5 2}$ as a potential substrate. The resulting cascade provided a straightforward access to a range of chiral cyclopropane spiro-oxindoles $53^{[38]}$ 
<smiles>[R]C=CC(=O)C=C([CH2+])[CH-]C([R])=O</smiles>

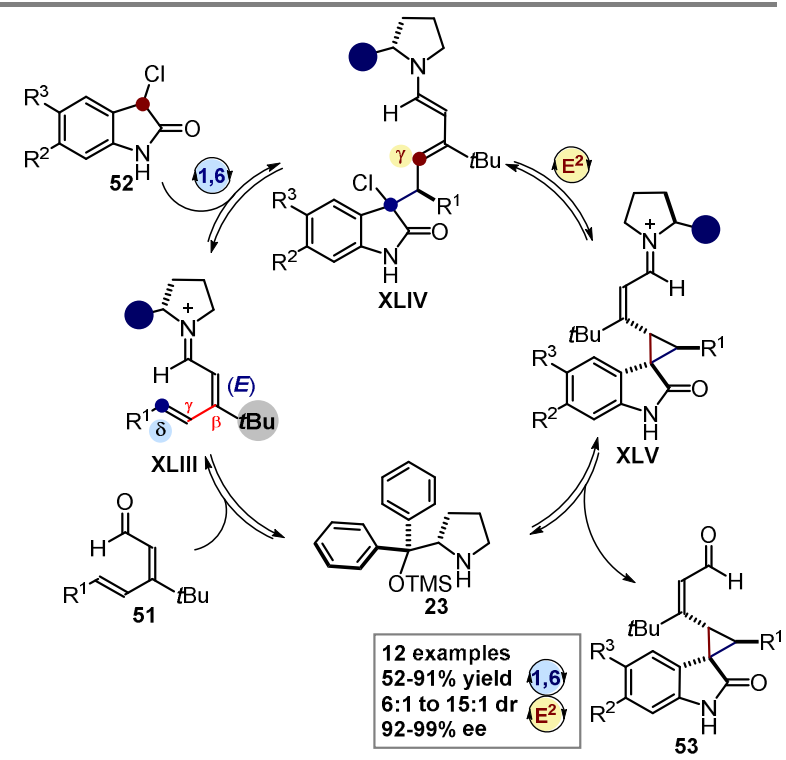

Scheme 16. Synthesis of cyclopropane spiro-oxindoles $\mathbf{5 3}$ by means of a 1,6$E^{2}$ cascade reaction with linear dienals; 1,6: vinylogous iminium ion; $E^{2}$ : dienamine. The blue circle represents the chiral scaffold of the aminocatalyst 23. The grey circle in XLIII highlights the importance of the tert-butyl moiety as a steering group.

The reaction relies on the activation of the extended dienal 51 by means of vinylogous iminium ion formation XLIII. The presence of a bulky tert-butyl substituent at the $\beta$-position was essential to secure high levels of both regio- and stereoselectivity. Specifically, the tert-butyl group, serving as a steering group, directed the nucleophilic attack of $\mathbf{5 2}$ to the less congested $\delta$-carbon while controlling the molecular topology of the extended iminium ion XLIII. NMR spectroscopic studies indicated that the dominant ground-state conformer in solution has an $E, E, E$ topology, with the same configuration for the three double bonds in XLIII. The steric prominence of the tert-butyl group makes it a topologically dominant element that can enforce an uncommon $s$-cis conformation around the single $C(\beta)-C(\gamma)$ bond (highlighted in red in XLIII, Scheme 16). Collectively, these features contribute to a highly preorganized, configurationally stable, transient intermediate XLIII, which was crucial to reaction development. Indeed, the chiral fragment within the catalyst $\mathbf{2 3}$ was positioned close enough to the reactive $\delta$-carbon to determine an effective shielding of the $R e$ face of the extended iminium ion XLIII, leaving the opposite Si face available for the approach of the 2chlorooxindole 52. The resulting dienamine intermediate XLIV then underwent an intramolecular nucleophilic substitution affording, after hydrolysis, the cyclopropane spiro-oxindole $\mathbf{5 3}$ in good yields and high levels of stereocontrol.

The next step in further expanding the versatility of vinylogous iminium ion-initiated cascades was to overcome the need for carefully designed substrates. Indeed, the first step of the previously developed $1,6-E^{2}$ sequences all required the generation of a multifunctional intermediate able to successively undergo an intramolecular process. The possibility of using linear polyunsaturated carbonyl compounds, though, provided a more flexible tool for designing more complicated cascade reactions based on intermolecular and stereoselective bond-forming events. Specifically, a three-component domino process proceeding by way of an aminocatalyzed Michael addition/1,6addition/vinylogous aldolization sequence, which combines two intermolecular and one intramolecular bond-forming event, was developed. The triple vinylogous organocascade yielded valuable spiro-oxindolic cyclohexane derivatives $\mathbf{5 7}$ with six stereogenic centers and very high control over the stereochemistry (from 4:1 to $17: 1 \mathrm{dr}, 99 \%$ ee, Scheme 17$){ }^{[39,40]}$
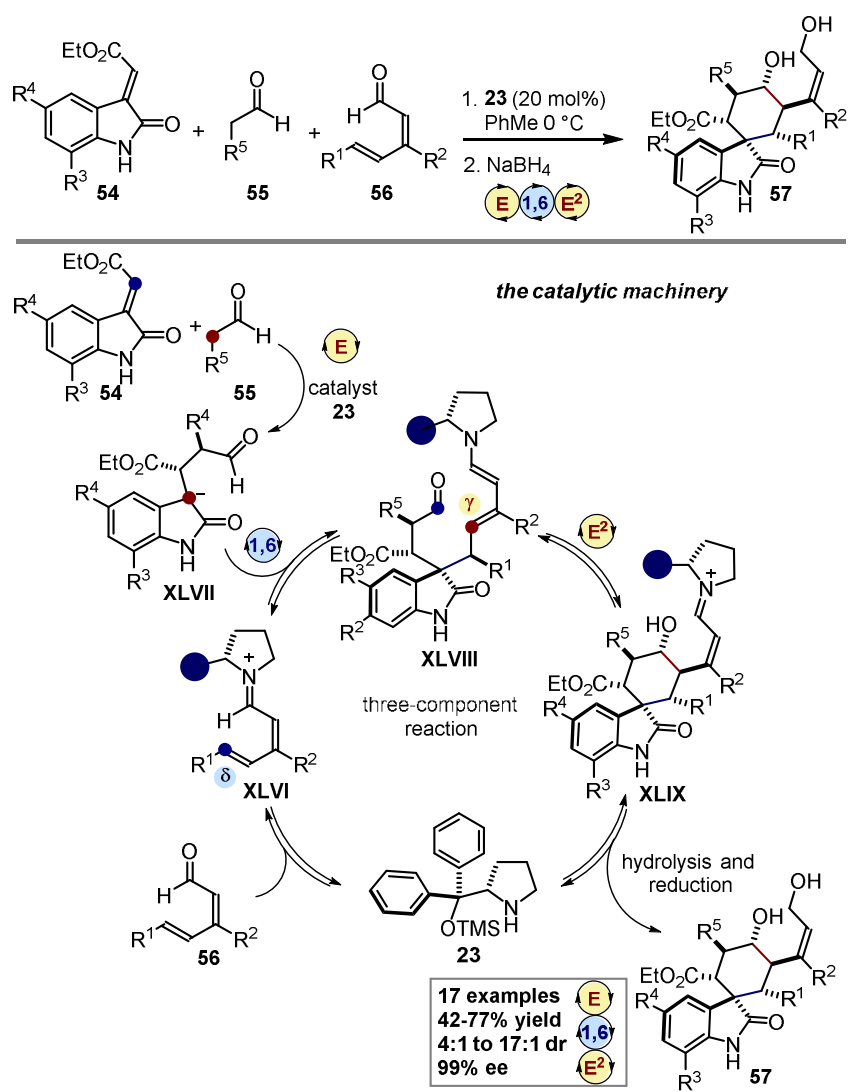

Scheme 17. The vinylogous triple cascade proceeds by way of an enamine $(E) /$ vinylogous iminium ion $(1,6) /$ dienamine activation $\left(E^{2}\right)$ sequence; $E$ : enamine; 1,6 : vinylogous iminium ion; $E^{2}$ : dienamine. The blue circle represents the chiral scaffold of the aminocatalyst 23 . The reactive carbon atoms are highlighted in different colors depending on their inherent reactivity: magenta stands for a nucleophilic position, blue for an electrophilic center.

The triple cascade process is based on complicated catalytic machinery, which is largely based on vinylogous reactivity. An enamine-catalyzed Michael addition of the aldehyde $\mathbf{5 5}$ to the alkylidine oxindole acceptor $\mathbf{5 4}$ leads to the transient formation of the nucleophilic intermediate XLVII. The nucleophile can then engage in the $y$-site selective intermolecular 1,6-addition, upon vinylogous iminium ion activation of the $\beta$-substituted 2,4-dienal 
56, to forge the spiro-stereocenter. The transient formation of a nucleophilic dienamine $\mathbf{X L V I I}$ drives an intramolecular vinylogous aldolization to provide the final cyclohexane product 57 .

\subsection{Sequential Vinylogous Iminium Ion/Iminium Ion Cascade \\ Pathway}

Vinylogous iminium ion-initiated vinylogous cascade processes are not limited to the functionalization of $\alpha, \beta, \gamma, \delta$-unsaturated carbonyl compounds at the adjacent $\delta$ and $\gamma$ carbon atoms. Stereoselective functionalization of the $\delta$ - and $\beta$-carbons can also be accomplished when using a dual LUMO-lowering activation sequence, specifically a vinylogous iminium ion /iminium ion pathway (1,6/1,4 cascade, Figure 9). After condensation of the carbonyl compound with a chiral aminocatalyst, the resultant vinylogous iminium ion is intercepted by a nucleophile affording, in analogy to the precedent $1,6-\mathrm{E}^{2}$ sequence, a dienamine intermediate. At this point, protonation of the dienamine results in the formation of an iminium ion intermediate that can trap a second nucleophilic species. If the original substrate features a second nucleophilic site, an additional bond formation follows under the control of the chiral catalyst. This vinylogous iminium ion/iminium ion $(1,6-1,4)$ cascade reaction allows functionalization of a carbonyl at the $\delta$ - and $\beta$-positions and relies on the chiral aminocatalyst for both bond forming steps.

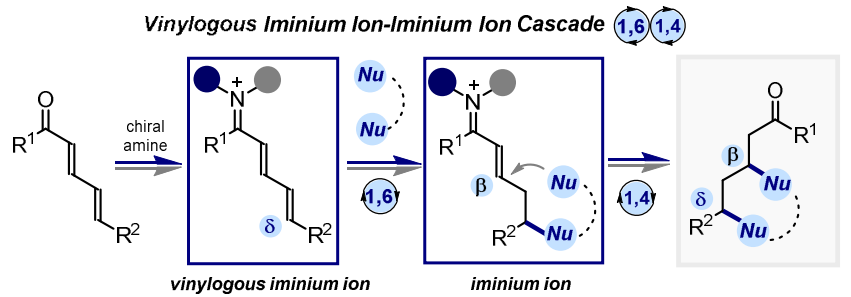

Figure 9. Vinylogous iminium ion $(1,6)$ /iminium ion $(1,4)$ cascade pathway; the blue and grey circles represent the chiral scaffolds of the aminocatalyst.

When planning a vinylogous iminium ion-initiated cascade reaction of a linear polyunsaturated carbonyl compound, the main difficulty is that the chiral catalyst must forge a remote stereocenter with high fidelity while inducing exclusive $\delta$-site selectivity in the initial 1,6-addition. This is strictly dependent on the ability of controlling the molecular topology of the acyclic intermediate, so as to ensure highly predictable reaction outcomes. As discussed in the precedent section, the use of the linear 2,4-dienals 56, bearing a tert-butyl steering group at the $\beta$ position, ensured molecular preorganization of the catalytically active vinylogous iminium ion intermediate (Scheme 18). The presence of such steric control element resulted in only the conformationally stable $E, E, E$ iminium ion isomer $\mathbf{L}$ forming, and this turned out to be key for achieving high selectivity. The use of 3-hydroxyoxindoles $\mathbf{5 8}$, which is characterized by a bidentate nucleophilic profile (both the $\mathrm{C} 3$ carbon and the oxygen atom are nucleophilic sites), allowed the realization of a 1,6/1,4 cascade with enals $56 .{ }^{[41]}$ This process, which is based on a highly regioand stereoselective 1,6-addition/oxa-Michael sequence, directly afforded tetrahydrofuran spirooxindole derivatives $\mathbf{5 9}$. Mechanistically, the reaction proceeds through the formation of the $E, E$ iminium ion isomer $\mathbf{L}$, which drives a selective 1,6 -addition of the nucleophilic carbon within $\mathbf{5 8}$ to form the iminium ion intermediate LI. Then, the hydroxyl group participates in an intramolecular oxa-Michael process leading to the final spirocyclic adduct 59

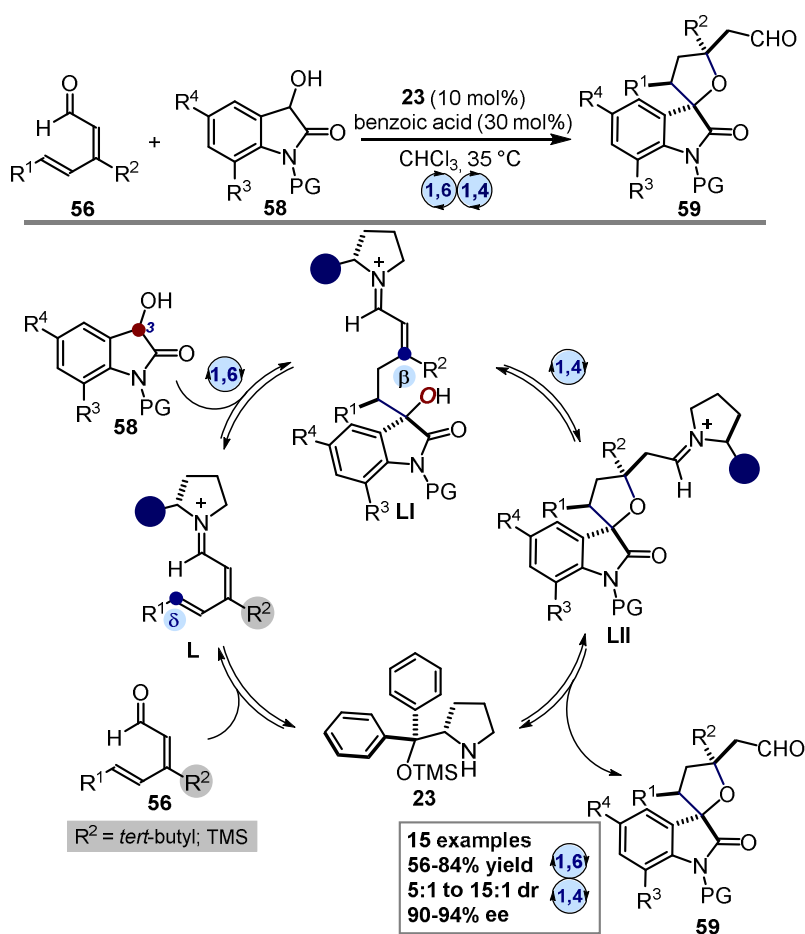

Scheme 18. Synthesis of tetrahydrofuranyl spirooxindoles 59 by means of a 1,6-1,4 cascade reaction with linear dienals; 1,6 : vinylogous iminium ion; 1,4: iminium ion. The blue circle represents the chiral scaffold of the aminocatalyst 23. The grey circle in $\mathbf{L}$ highlights the importance of the bulky $R^{2}$ substituent as a conformational anchor; TMS: trimethylsilyl; PG: protecting group. The reactive carbon atoms are highlighted in different colors depending on their inherent reactivity: magenta stands for a nucleophilic position, blue for an electrophilic center.

Remarkably, the presence at the $\beta$ position of the dienal substrate 56 of a trimethylsilyl group, characterized by a similar Charton steric parameter ${ }^{[42]}$ of a tert-butyl moiety, directed the process exclusively toward a 1,6-addition manifold while preserving the high enantioselectivity. This result further highlights how strongly the steric profile of the $\beta$-substituent in $\mathbf{5 6}$ is connected with an effective control over the molecular topology of the vinylogous iminium ion intermediate. Synthetically, it is worth noting that the trimethylsilyl group could be eventually removed upon protiodesilylation under basic conditions, which makes the trimethyl silyl moiety a traceless directing group to achieve $\delta$-site selectivity, providing a formal 1,6-addition of geometrically unbiased, linear dienals.

Recently, Jørgensen and coworkers reported that different types of naphthols 61 were suitable substrates for the 1,6-1,4 vinylogous cascade process with linear, completely unbiased enals 60 (Scheme 19). ${ }^{[4]}$ Mechanistically, a Friedel-Crafts 1,6addition reaction of hydroxyarenes to a vinylogous iminium ion intermediate LIII and subsequent oxa-Michael addition to the iminium ion LIV formed in the first step led to chromans $\mathbf{6 2}$ with high stereofidelity. Importantly, no substituents on the 2,4-dienal 60 are required to ensure complete remote selectivity in the first step. Computational studies suggested that the $\delta$-site selectivity 
is driven by thermodynamic control of the Friedel-Crafts reaction step.

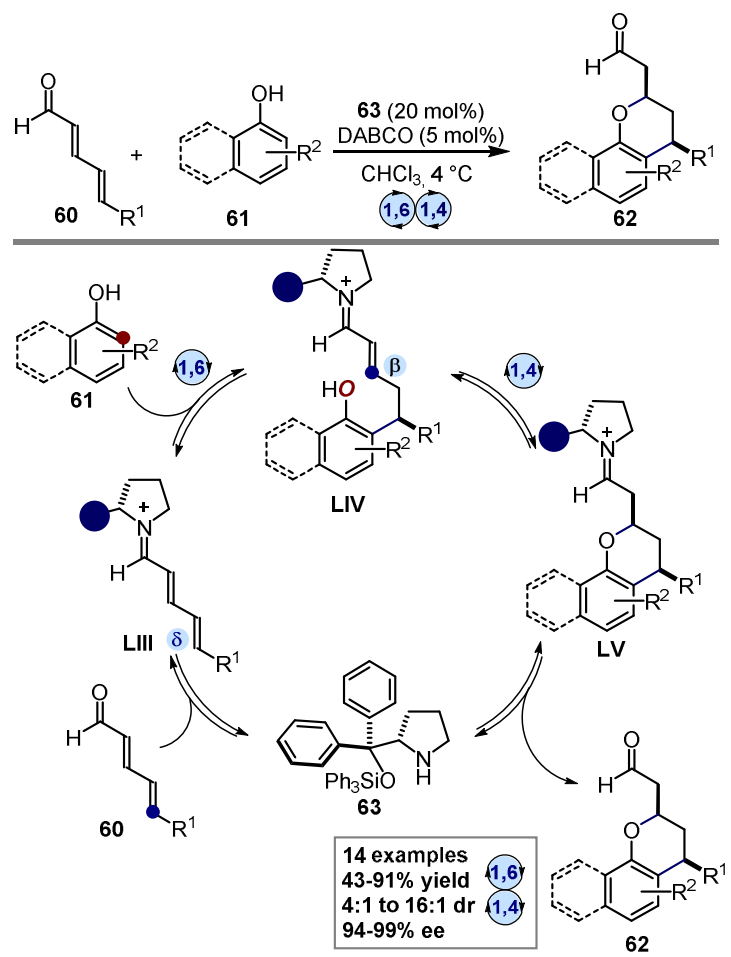

Scheme 19. 1,6-1,4 cascade reaction with linear, completely unbiased dienals; 1,6: vinylogous iminium ion; 1,4 : iminium ion. The blue circle represents the chiral scaffold of the aminocatalyst 63. The reactive carbon atoms are highlighted in different colors depending on their inherent reactivity: magenta stands for a nucleophilic position, blue for an electrophilic center.

\section{N-Heterocyclic Carbene-mediated Vinylogous Cascade} Processes

Aminocatalysis is not the only successful organocatalytic approach for designing vinylogous cascade reactions. $\mathrm{N}$ - heterocyclic carbenes $(\mathrm{NHC})^{[44]}$ have also been used as viable catalytic intermediates for these transformations. Evidence for the existence of carbenes dates back to the late $19^{\text {th }}$ century, ${ }^{[44 a]}$ and then they have been used extensively as organocatalysts to functionalize carbonyl compounds at the $\alpha$ - and $\beta$-positions. The activation modes that permit such reactivity can also be extended into the vinylogous reactivity space to facilitate remote transformations, including stepwise cascade processes.

Three strategies have been reported to facilitate NHC-catalyzed vinylogous cascade processes (Figure 10). The first two are related and proceed through a common activated ester intermediate. This intermediate can be formed either through the addition of an $\mathrm{NHC}$ to an enal and the subsequent oxidation of the Breslow intermediate (Strategy 1 ) or through the addition of an $\mathrm{NHC}$ to an $\alpha, \beta$-unsaturated ester to directly access the activated ester intermediate (Strategy 2). This strategy results in the activation of the remote $\mathrm{y}$-carbon which, in the presence of a base, undergoes deprotonation to afford a vinylogous enolate. A third conceptually different strategy has also been reported, which requires enals that feature a leaving group at the $y$-position. Following the addition of the $\mathrm{NHC}$, intramolecular elimination from the Breslow intermediate generates the crucial vinylogous enolate species (Strategy 3 in Figure 11).

Once the vinylogous enolate has been formed, it can undergo a vinylogous nucleophilic attack to an electrophile. If this electrophile is tethered to a masked nucleophile, a cascade reaction occurs where the nucleophilic moiety utilizes the intrinsic nature of the $\mathrm{NHC}$ as a leaving group to attack the carbonyl moiety and undergo a cyclization $\left(E n^{2}-S\right)$ (Figure 11). These $E n^{2}-$ $\mathrm{S}$ cascade reactions rely on the NHC catalyst for both new bond forming steps and, although a cycloaddition pathway cannot be excluded for these types of transformations, the current understanding is that they are more likely to proceed through a cascade process. ${ }^{[45]}$

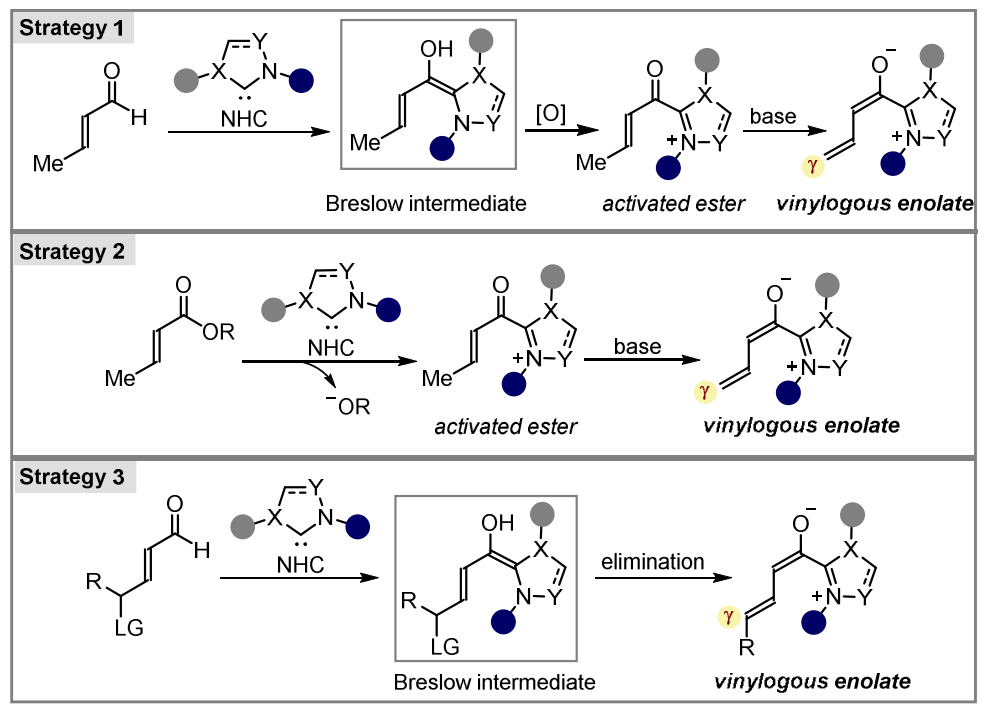

Figure 10. The different strategies utilized to form vinylogous enolates using NHC catalysis; the blue and grey circles represent the chiral scaffold of the NHC catalyst. 


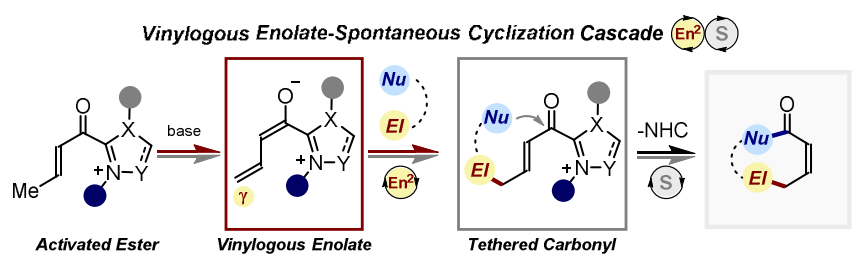

Figure 11. Vinylogous enolate $\left(E n^{2}\right) / s p o n t a n e o u s(S)$ cyclization cascade pathway; the initial organocatalytic event is followed by a spontaneous ipsocyclization. The blue and grey circles represent the chiral scaffolds of the NHC catalyst.

The first reported use of NHC-catalysis to develop a $\mathrm{En}^{2}-\mathrm{S}$ vinylogous cascade reaction was based on Strategy 1 to activate the $\mathrm{y}$-carbon. This work, shown in Scheme 20, featured the formation of lactones 66 from enals 64 and electron-deficient ketones $65{ }^{[46]}$ In the mechanistic proposal, the addition of the $\mathrm{NHC} \mathbf{6 7}$ to enal $\mathbf{6 4}$ forms the Breslow intermediate LVI that, upon oxidation from 68, generates intermediate LVII. Deprotonation of LVII affords the vinylogous enolate LVIII which undergoes a Lewis acid-mediated vinylogous addition to the ketone 65 . After this addition, the resulting anionic intermediate LIX undergoes a spontaneous intramolecular cyclization to form the lactone product 66 in good yields (52-82\%) and enantioselectivities (60$94 \%$ ee)

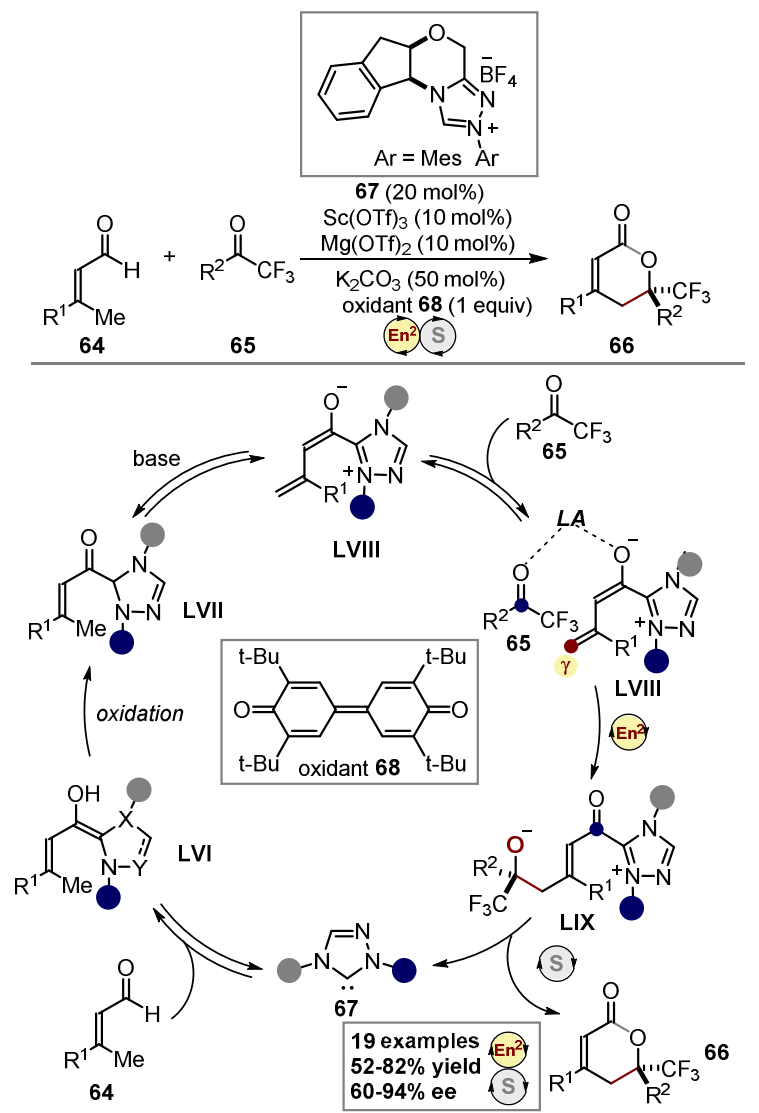

Scheme 20. The first reported use of NHC catalysis to develop a vinylogous cascade; $\mathrm{En}^{2} / \mathrm{S}$ cyclization pathway. $\mathrm{En}^{2}$ : vinylogous enolate, S: spontaneous cyclization. The blue and grey circles represent the chiral scaffolds of the NHC catalyst 67 . The reactive carbon atoms are highlighted in different colors depending on their inherent reactivity: magenta stands for a nucleophilic position, blue for an electrophilic center.

Strategy 1 was also used for the formation of bicycle $\mathbf{7 0}$ through a formal [3+4] cycloaddition between enal 64 and azomethine 69 in the presence of NHC 67 and the oxidant 68 (Scheme 21). ${ }^{[47]}$ The mechanism is believed to proceed similarly to the one illustrated in Scheme 20: the vinylogous enolate LVIII, upon nucleophilic addition to imine 69, forms the [3+4] adduct 70 after spontaneous intramolecular lactamization in good yields (51$81 \%$ ) and enantioselectivities (84-99\% ee)

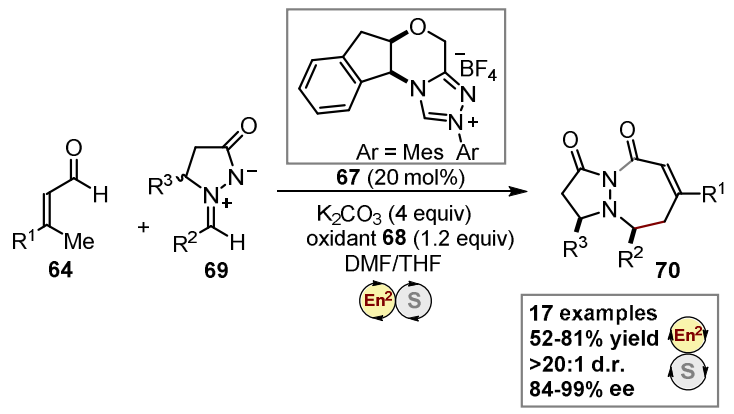

Scheme 21. The formation of the formal [4+3] product 70 through a $E n^{2}-S$ cascade sequence; $\mathrm{En}^{2} / \mathrm{S}$ cyclization pathway; $\mathrm{En}^{2}$ : vinylogous enolate, S: spontaneous cyclization

The use of Strategy 2 to form the vinylogous enolate (Figure 10) has also served for the implementation of a $\mathrm{En}^{2}-\mathrm{S}$ cascade sequence. Direct addition of NHC 74 to the $\alpha, \beta$-unsaturated ester 71 generates the intermediate without the need for an oxidation step. Besides the different activation strategy to access the key vinylogous enolate intermediate, the reaction proceeds in a similar fashion as discussed in Scheme 20: vinyl enolate LVIII attacks the hydrazine $\mathbf{7 2}$ and then a spontaneous cyclization delivered the lactam product $\mathbf{7 3}$ in good yields (31-91\%) and enantiomeric excess $\left(90-99 \%\right.$ ee).$^{[48]}$

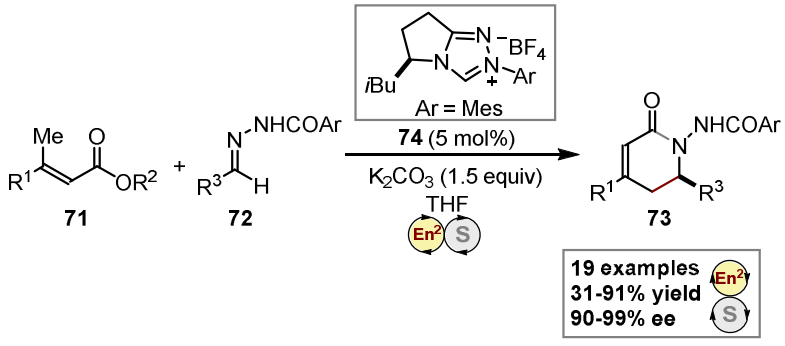

Scheme 22. NHC-mediated $E n^{2}-S$ cascade sequence; En²: vinylogous enolate, $\mathrm{S}$ : spontaneous cyclization. Formation of the vinylogous enolate achieved via Strategy 2 in Figure 10.

Recently, the vinylogous enolate-generation Strategy 3 (Figure 10) was also applied to realize an $E^{2}-S$ cascade sequence. Using the enal $\mathbf{7 5}$, which bears a suitable carbonate leaving group at the y position, it was possible to generate the intermediate LVIII after elimination of the leaving group following formation of the Breslow intermediate LX. Nucleophilic addition of LVIII to di-tertbutyl azodicarboxylate $\mathbf{7 6}$ and subsequent lactamization gave product 77 in good yields (49-82\%) and enantiomeric excess (94$99 \%$ ee, Scheme 23$){ }^{[49]}$ 

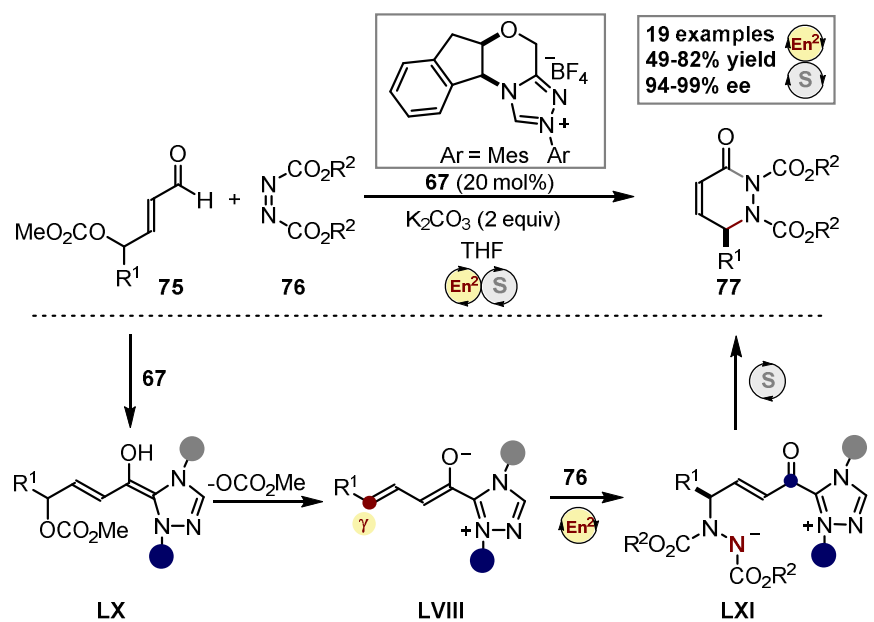

Scheme 23. NHC-mediated $\mathrm{En}^{2}-\mathrm{S}$ cascade sequence; $\mathrm{En}^{2}$ : vinylogous enolate, $\mathrm{S}$ : spontaneous cyclization. Formation of the vinylogous enolate achieved via Strategy 3 in Figure 10.

\section{Summary and Outlook}

The intense investigations into organocatalytic cascade reactions have led to the development of highly innovative techniques, which now provide reliable and versatile tools for modern asymmetric synthesis. Recently, the combination of organocatalysis and the principle of vinylogy has opened up new avenues for reaction design, further expanding the synthetic potential of cascade processes. Vinylogous cascade strategies provide effective means to build stereochemically dense, complex molecules from readily available substrates and using simple producers while forging stereogenic centers remote from the catalyst's point of action. Further interesting development along these lines are foreseen in the near future. ${ }^{[50]}$

Hamish B. Hepburn was born in Dundee, Scotland (1988) and completed his undergraduate studies at the University of Edinburgh, UK (2006-2011). He then stayed at the same institution and completed his $\mathrm{PhD}$ in the field of rhodium catalysis under the supervision of Professor Hon Wai Lam (2011-2014, including one year at the University of Nottingham, UK). In January 2015 he joined the group of Paolo Melchiorre at ICIQ, Spain where he is currently a Marie Curie COFUND - Severo Ochoa postdoctoral fellow, investigating organocatalytic photochemical reactions.

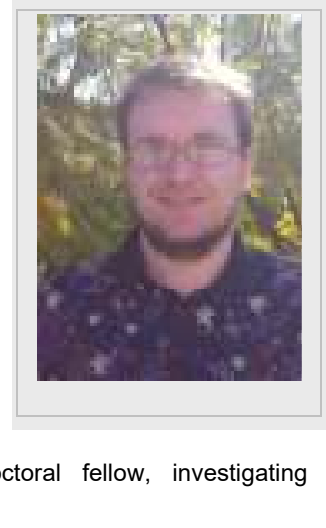

Luca Dell'Amico was born in Carrara, Italy. In 2010 he completed his M. Sc. in Medicinal Chemistry at the Parma University (I), where, in the same year, he started his PhD. In 2013 he spent a period of research at the "Center for Catalysis" at Århus Univesity (Denmark), working with Prof. Karl Anker Jørgensen. Luca finished his $\mathrm{PhD}$ (Doctor Europaeus degree) at the Parma University in 2014 under the supervision of Prof. Franca Zanardi. In 2014 he moved to the ICIQ

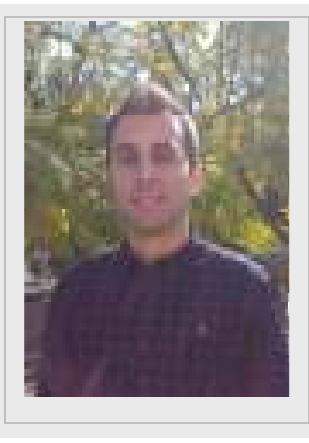

(Spain), joining the group of Prof. Paolo Melchiorre. Luca is currently a Marie Curie COFUND - postdoctoral fellow, and he is investigating novel organocatalytic photochemical vinylogous reactivity.

Paolo Melchiorre was born in 1973 in Camerino (Italy). He earned his M.Sc. degree (1999) and then his $\mathrm{PhD}$ in Chemistry (2003) from Bologna University under the supervision of A. Umani-Ronchi and P. G. Cozzi. After a research period with K. A. Jørgensen at Center for Catalysis, Århus University (Denmark), he joined the research group of $\mathrm{G}$. Bartoli at Bologna University, where he became Assistant Professor in 2007. In 2009, Paolo moved to

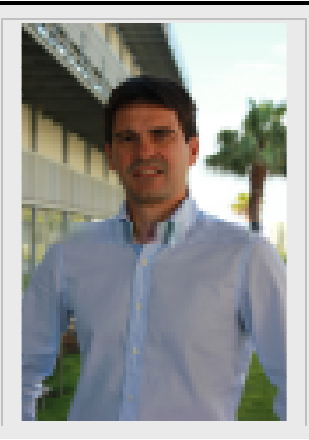

Tarragona (Spain) as an ICREA Research Professor and an ICIQ Senior Group Leader. His current scientific interests lie on the discovery and mechanistic elucidation of new enantioselective organocatalytic and photochemical processes.

\section{Acknowledgements}

Funding for this work was provided by the Institute of Chemical Research of Catalonia (ICIQ) Foundation and the European Research Council (ERC Starting grant agreement no. 278541 ORGA-NAUT). L.D. and H.B.H. thank the Marie Curie COFUND action (2014-1-ICIQ-IPMP) and Severo Ochoa Excellence Accreditation 2014-2018, SEV-2013-0319 for postdoctoral fellowships.

Keywords: aminocatalysis • cascade catalysis • organocatalysis - molecular complexity $\cdot$ vinylogous reactivity 


\section{References}

[1] a) K. C. Nicolaou, D. J. Edmonds, P. G. Bulger, Angew. Chem. Int. Ed 2006, 45, 7134-7186; b) C. J. Chapman, C. G. Frost, Synthesis 2007 1-21; c) K. C. Nicolaou, J. S. Chen, Chem. Soc. Rev. 2009, 38, 29933009; d) Catalytic Cascade Reactions, P.-F. Xu, W. Wang (Eds), WileyVCH, Weinheim, 2013.

[2] a) L. F. Tietze, U. Beifuss, Angew. Chem. Int. Ed. Engl. 1993, 32, 131 163; b) Domino Reactions in Organic Synthesis, L. F. Tietze, G. Brasche K. Gericke (Eds), Wiley-VCH, Weinheim, 2006.

[3] Chemoselectivity lies at the heart of modern organic chemistry. For an inspiring conspectus on the topic, see: a) R. A. Shenvi, D. P. O'Malley, P. S. Baran, Acc. Chem. Res. 2009, 42, 530-541. For an overview on how to achieve chemoselectivity in aminocatalytic domino strategies, see: b) M. Marigo, P. Melchiorre, ChemCatChem 2010, 2, 621-623.

[4] a) D. J. Ramón, M. Yus, Angew. Chem. Int. Ed. 2005, 44, 1602-1634; b) A. M. Walji, D. W. C. MacMillan, Synlett 2007, 1477-1489.

[5] The Horeau principle provides the mathematical foundation for rationalizing the enantioenrichment observed along successive catalytic cycles of asymmetric cascade processes, see: a) J. P. Vigneron, M. Dhaenens, A. Horeau, Tetrahedron 1973, 29, 1055-1059. For a nice application in asymmetric catalysis, see: b) J. A. Enquist Jr., B. M. Stoltz, Nature 2008, 453, 1228-1231.

[6] a) D. W. C. MacMillan, Nature 2008, 455, 304-308; b) E. N. Jacobsen, D. W. C. MacMillan, Proc. Natl. Acad. Sci. U.S.A. 2010, 107, 2061820619.

[7] For reviews, see: a) D. Enders, C. Grondal, M. R. M. Hüttl, Angew. Chem. Int. Ed. 2007, 46, 1570-1581; b) H. Pellissier, Adv. Synth. Catal. 2012 354, 237-294. Recently, a general classification and nomenclatura system for systematically and informatively describing each one-pot reaction was introduced, see: c) Ł. Albrecht, H. Jiang, K. A. Jørgensen, Angew. Chem. Int. Ed. 2011, 50, 8492-8509.

[8] For overviews on applications to the total synthesis of natural products, see: a) C. Grondal, M. Jeanty, D. Enders, Nature Chem. 2010, 2, $167-$ 178. For an outstanding example of the potential for aminocatalytic cascade processes to access complex frameworks useful to streamline the synthesis of natural products, see: b) S. B. Jones, B. Simmons, A Mastracchio, and D. W. C. MacMillan, Nature 2011, 475, 183-188.

[9] For seminal papers, see: a) U. Eder, G. Sauer, R. Wiechert, Angew. Chem. Int. Ed. Engl. 1971, 10, 496-497; b) Z. G. Hajos, D. R. Parrish, J. Org. Chem. 1974, 39, 1615-1621; c) B. List, R. A. Lerner, C. F. Barbas III, J. Am. Chem. Soc. 2000, 122, 2395-2396.

[10] K. A. Ahrendt, C. J. Borths, D. W. C. MacMillan, J. Am. Chem. Soc. 2000, 122, 4243-4244.

[11] For insightful perspectives on the historical origins of aminocatalysis, see: a) B. List, Angew. Chem. Int. Ed. 2010, 49, 1730-1734; b) C. F. Barbas III, Angew. Chem. Int. Ed. 2008, 47, 42-47; c) G. Lelais, D. W. C MacMillan, Aldrichimica Acta 2006, 39, 79-87.

[12] For the pioneering reports which established the possibility of integrating iminium ion and enamine activation modes into more elaborate reaction sequences, see: a) Y. Huang, A. M. Walji, C. H. Larsen, D. W. C. MacMillan, J. Am. Chem. Soc. 2005, 127, 15051-15053; b) M. Marigo, T. Schulte, J. Franzen, K. A. Jørgensen, J. Am. Chem. Soc. 2005, 127 15710-15711. For another landmark report, see: c) D. Enders, M. R. M. Hüttl, C. Grondal, G. Raabe, Nature 2006, 441, 861-863.

[13] HOMO- and LUMO-activations are general and fundamental concepts of reactivity, which permeate the whole area of asymmetric catalysis, e.g. LUMO-activation is the principle behind the classical Lewis-acid activation of a carbonyl compound by a metal salt.

[14] The Principle of Vinylogy accounts for the transmission of electronic effects through a conjugated $\pi$-system, as defined a long time ago: $R$. C. Fuson, Chem. Rev. 1935, 16, 1-27.

[15] For a review highlighting the difficulties of controlling remote stereocenters, see: J. Clayden, Chem. Soc. Rev. 2009, 38, 817-829.
[16] For reviews on vinylogous organocatalytic strategies for achieving remote stereocontrol, see: a) E. Arceo, P. Melchiorre, Angew. Chem. Int Ed. 2012, 51, 5290-5292; b) H. Jiang, L. Albrecht, K. A. Jørgensen, Chem. Sci. 2013, 4, 2287-2300; c) I. D. Jurberg, I. Chatterjee, R. Tannert, P. Melchiorre, Chem. Commun. 2013, 49, 4869-4883.

[17] For early works involving dienamine catalysis, see: a) $S$. Bertelsen, $M$ Marigo, S. Brandes, P. Dinér, K. A. Jørgensen, J. Am. Chem. Soc. 2006 128, 12973-12980; b) B. J. Bench, C. Liu, C. R. Evett, C. M. H. Watanabe, J. Org. Chem. 2006, 71, 9458-9463. For a review on dienamine activation, see: c) D. B. Ramachary, Y. V. Reddy, Eur. J. Org. Chem. 2012, 865-887.

[18] For early examples involving trienamine catalysis, see: a) Z.-J. Jia, H Jiang, J.-L. Li, B. Gschwend, Q.-Z. Li, X. Yin, J. Grouleff, Y. C. Chen, K. A. Jørgensen, J. Am. Chem. Soc. 2011, 133, 5053-5061; b) Z.-J. Jia, Q. Zhou, Q.-Q. Zhou, P.-Q. Chen, Y.-C. Chen, Angew. Chem. Int. Ed. 2011, 50, 8638-8641; c) Y. Liu, M. Nappi, E. Arceo, S. Vera, P. Melchiorre, J. Am. Chem. Soc. 2011, 133, 15212-15218. For a review on trienamine activation, see: d) I. Kumar, P. Ramaraju, N. A. Mir, Org. Biomol. Chem. 2013, 11, 709-716.

[19] For an early example, see: a) X. Tian, Y. K. Liu, P. Melchiorre, Angew. Chem. Int. Ed. 2012, 51, 6439-6442. For a highlight article: b) M.J. Lear, Y. Hayashi, ChemCatChem 2013, 5, 3499-3501.

[20] a) K. Liu, A. Chougnet, W.-D. Woggon, Angew. Chem. Int. Ed. 2008, 47, 5827-5829. Early reports by the group of Hong described a potential vinylogous cascade reaction using a dienamine-iminium ion activation sequence; however, the reactions gave a mixture of products and the mechanism remained not well understood, see: b) B.-C. Hong, M.-F. Wu, H.-C. Tseng, J.-H. Liao, Org. Lett. 2006, 8, 2217-2220.

[21] a) K. Liu, W.-D. Woggon, Eur. J. Org. Chem. 2010, 1033-1036, b) M. C Bröhmer, E. Bourcet, M. Nieger, S. Bräse. Chem. Eur. J. 2011, 17 13706-13711; c) K. Liu, X. Jiang, Eur. J. Org. Chem. 2015, 6423-6428.

[22] $\quad$. Albrecht, G. Dickmeiss, C. F. Weise, C. Rodriguez-Escrich, K. A. Jørgensen, Angew. Chem. Int. Ed. 2012, 51, 13109-13113.

[23] t. Albrecht, G. Dickmeiss, F. Cruz Acosta, C. Rodriguez-Escrich, R. L. Davis, K. A. Jørgensen, J. Am. Chem. Soc. 2012, 134, 2543-2546.

[24] G. Talavera, E. Reyes, J. L. Vicario, L. Carrillo, Angew. Chem. Int. Ed. 2012, 51, 4104-4107.

[25] W. Xiao, X. Yin, Z. Zhou, W. Du, Y.-C. Chen, Org. Lett. 2016, 18, 116119.

[26] J.-L. Li, S.-L. Zhou, P.-Q. Chen, L. Dong, T.-Y. Liu, Y.-C. Chen, Chem. Sci, 2012, 3, 1879-1882

[27] J. Stiller, P. H. Poulsen, D. Cruz Cruz, J. Dourado, R. L. Davis, K. A Jørgensen, Chem. Sci. 2014, 5, 2052-2056.

[28] B. S Donslund, K. Søholm Halskov, L. A. Leth, B. Matos Paz, B.; K. A. Jørgensen, Chem. Commun. 2014, 50, 13676-13679.

[29] L. K. Ransborg, M. Overgaard, J. Hejmanowska, S. Barfüsser, K. A. Jørgensen, Ł. Albrecht, Org. Lett. 2014, 16, 4182-4185.

[30] X. Feng, Z. Zhou, C. Ma, X. Yin, R. Li, L. Dong, Y.-C. Chen, Angew. Chem. Int. Ed. 2013, 52, 14173-14176.

[31] For a review on the use of the cinchona-based primary amines in enantioselective organocatalysis, see: P. Melchiorre, Angew. Chem. Int. Ed. 2012, 51, 9748-9770.

[32] Y. Li, F. Tur, R. P. Nielsen, H. Jiang, F. Jensen, K. A. Jørgensen, Angew. Chem. Int. Ed. 2016, 55, 1020-1024.

[33] J. Gu, B.-X. Xiao, Y.-R. Chen, W. Du, Y.-C. Chen, Adv. Synth. Catal. 2016, 358, 296-302.

[34] a) B. Tan, N. R. Candeias, C. F. Barbas, III Nat. Chem. 2011, 3, 473477; b) K. Albertshofer, B. Tan, C. F. Barbas, III. Org. Lett. 2012, 14, 1834-1837.

[35] X. Tian, P. Melchiorre, Angew. Chem. Int. Ed. 2013, 52, 5360-5363.

[36] X. Gu, T. Guo, Y. Dai, A. Franchino, J. Fei, C. Zou, D. J. Dixon, J. Ye, Angew. Chem. Int. Ed. 2015, 54, 10249-10353.

[37] K. Halskov, T. Naicker, M. E. Jensen. K. A. Jørgensen Chem. Comm 2013, 49, 6382-3684. 
[38] R. C. da Silva I. Chatterjee, E. Escudero-Adán, M. Weber Paixão, P. Melchiorre, Asian J. Org. Chem. 2014, 3, 466-469.

[39] I. Chatterjee, D. Bastida, P. Melchiorre, Adv. Synth. Catal. 2013, 355, 3124-3130.

[40] We note that this reaction is not a true "cascade reaction" as not all the reagents are present at the commencement of the reaction; indeed, the 2,4-dienal $\mathbf{5 6}$ is added after 60 minutes from the start of the cascade. This vinylogous triple cascade reaction was largely inspired by the process originally developed by Dieter Enders, see Ref. [12c]. This process is recognized as a spectacular example of the potential of secondary amine-mediated multicomponent reactions to target stereochemical and molecular complexity.

[41] M. Silvi, I. Chatterjee Y. Liu, P. Melchiorre, Angew. Chem. Int. Ed. 2013, 52, 10780-10783.

[42] Charton values $(v): t$-Bu, $v=1.24 ;(\mathrm{Me})_{3} \mathrm{Si}, v=1.40$. a) M. Charton, $J$. Am. Chem. Soc. 1975, 97, 1552-1556; b) M. Charton, J. Am. Chem. Soc. 1975, 97, 3691-3693

[43] P. H. Poulsen, K. S. Feu, B. M. Paz, F. Jensen, K. A. Jørgensen, Angew. Chem. Int. Ed. 2015, 54, 8203-8207.

[44] a) E. Buchner, T. Curtius, Ber. Dtsch. Chem. Ges. 1885, 18, 2377-2379; b) H. Staudinger, O. Kupfer, Ber. Dtsch. Chem. Ges.1912, 45, 501-509; c) R. Breslow, J. Am. Chem. Soc. 1958, 80, 3719-3726. For a recent review: d) D. Enders, A. Henseler, Chem. Rev. 2007, 107, 5606-5655.
[45] Similar enantioselective catalytic cyclizations were achieved using Cinchona-based nucleophilic catalysts. However, in the original studies, the mechanism is discussed as a concerted cycloaddition pathway. a) $\mathrm{P}$. S. Tiseni, R. Peters, Angew. Chem. Int. Ed. 2007, 46, 5325-5328. b) P S. Tiseni, R. Peters, Chem. Eur. J. 2010, 16, 2503-2517. c) L.-T. Shen, L.-H. Sun, S. Ye. J. Am. Chem. Soc. 2011, 133, 15894-15897.

[46] J. Mo, X. Chen, Y. R. Chi, J. Am. Chem. Soc. 2012, 134, 8810-8813.

[47] M. Wang, Z. Huang, J. Xu, Y. R. Chi, J. Am. Chem. Soc. 2014, 136, 1214-1217.

[48] J. Xu, Z. Jin, Y. R. Chi, Org. Lett. 2013, 15, 5028-5031.

[49] X.-Y. Chen, F. Xia, J.-T. Cheng, S. Ye, Angew. Chem. Int. Ed. 2013, 52, 10644-10647.

[50] Following submission of this article, a remarkable example of vinylogous cascade sequence was reported. This transformation can be categorized as a 1,6-E-S triple cascade process, see: N. Hammer, L. A. Leith, J. Stiller, M. E. Jensen, K. A. Jørgensen, Chem. Sci. 2016, DOI: 10.1039/C6SC00185H. 


\section{PERSONAL ACCOUNT}

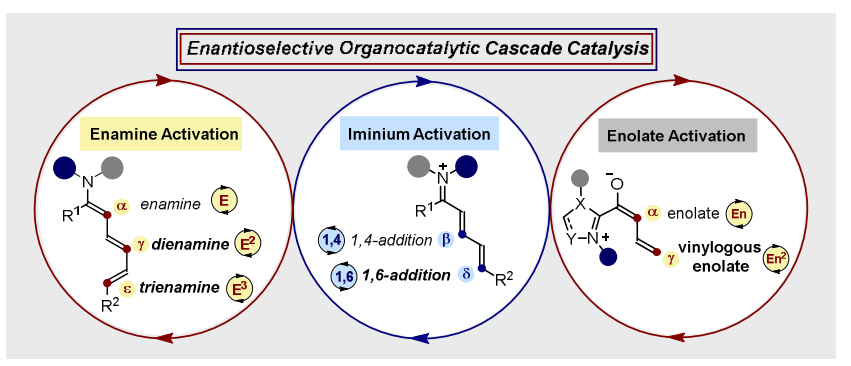

Hamish B. Hepburn, Luca Dell'Amico, and Paolo Melchiorre*

Page No. - Page No.

Enantioselective Vinylogous

Organocascade Reactions

This article critically describes the developments achieved in the field of enantioselective vinylogous organocascade reactions, charting the conceptual advances, and the milestone reactions that have been essential for reaching highly practical levels of synthetic efficiency. 